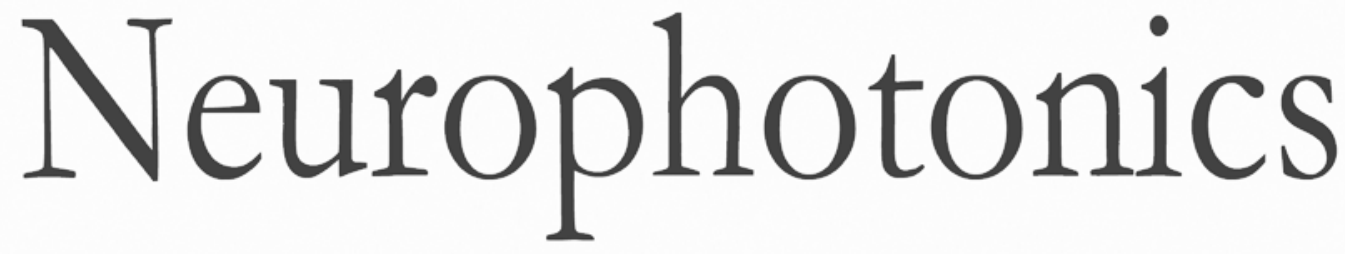

\title{
Quantification of the severity of hypoxic-ischemic brain injury in a neonatal preclinical model using measurements of cytochrome-c- oxidase from a miniature broadband- near-infrared spectroscopy system
}

Pardis Kaynezhad

Subhabrata Mitra

Gemma Bale

Cornelius Bauer

Ingran Lingam

Christopher Meehan

Adnan Avdic-Belltheus

Kathryn A. Martinello

Alan Bainbridge

Nicola J. Robertson

Ilias Tachtsidis 


\title{
Quantification of the severity of hypoxic-ischemic brain injury in a neonatal preclinical model using measurements of cytochrome-c-oxidase from a miniature broadband-near-infrared spectroscopy system
}

\author{
Pardis Kaynezhad, ${ }^{\mathrm{a}, \star}$ Subhabrata Mitra, ${ }^{\mathrm{b}}$ Gemma Bale, ${ }^{\mathrm{a}}$ Cornelius Bauer, ${ }^{\mathrm{a}}$ Ingran Lingam, ${ }^{\mathrm{b}}$ Christopher Meehan, \\ Adnan Avdic-Belltheus, ${ }^{b}$ Kathryn A. Martinello, ${ }^{b}$ Alan Bainbridge, ${ }^{c}$ Nicola J. Robertson, ${ }^{b}$ and Ilias Tachtsidis ${ }^{a}$ \\ aUniversity College London, Department of Medical Physics and Biomedical Engineering, London, United Kingdom \\ 'University College London, Institute for Women's Health, London, United Kingdom \\ 'University College London Hospital, Department of Medical Physics and Biomedical Engineering, London, United Kingdom
}

\begin{abstract}
We describe the development of a miniaturized broadband near-infrared spectroscopy system (bNIRS), which measures changes in cerebral tissue oxyhemoglobin $\left(\left[\mathrm{HbO}_{2}\right]\right)$ and deoxyhemoglobin $([\mathrm{HHb}])$ plus tissue metabolism via changes in the oxidation state of cytochrome-c-oxidase ([0xCCO]). The system is based on a small light source and a customized mini-spectrometer. We assessed the instrument in a preclinical study in 27 newborn piglets undergoing transient cerebral hypoxia-ischemia $(\mathrm{HI})$. We aimed to quantify the recovery of the $\mathrm{HI}$ insult and estimate the severity of the injury. The recovery in brain oxygenation $(\Delta[\mathrm{HbDiff}]=$ $\left.\Delta\left[\mathrm{HbO}_{2}\right]-\Delta[\mathrm{HHb}]\right)$, blood volume $\left(\Delta[\mathrm{HbT}]=\Delta\left[\mathrm{HbO}_{2}\right]+\Delta[\mathrm{HHb}]\right)$, and metabolism $(\Delta[\mathrm{oxCCO}])$ for up to $30 \mathrm{~min}$ after the end of $\mathrm{HI}$ were quantified in percentages using the recovery fraction (RF) algorithm, which quantifies the recovery of a signal with respect to baseline. The receiver operating characteristic analysis was performed on bNIRS-RF measurements compared to proton $\left({ }^{1} \mathrm{H}\right)$ magnetic resonance spectroscopic (MRS)-derived thalamic lactate/N-acetylaspartate (Lac/NAA) measured at 24-h post $\mathrm{HI}$ insult; Lac/NAA peak area ratio is an accurate surrogate marker of neurodevelopmental outcome in babies with neonatal $\mathrm{HI}$ encephalopathy. The $\Delta[\mathrm{oxCCO}]-$ RF cut-off threshold of $79 \%$ within 30 min of HI predicted injury severity based on Lac/NAA with high sensitivity $(100 \%)$ and specificity (93\%). A significant difference in thalamic Lac/NAA was noticed $(p<0.0001)$ between the two groups based on this cut-off threshold of $79 \% \Delta$ [oxCCO]-RF. The severe injury group $(n=13)$ had $\sim 30 \%$ smaller recovery in $\Delta[\mathrm{HbDiff}]-\mathrm{RF}(p=0.0001)$ and no significant difference was observed in $\Delta[\mathrm{HbT}]-\mathrm{RF}$ between groups. At $48 \mathrm{~h}$ post $\mathrm{HI}$, significantly higher ${ }^{31} \mathrm{P}$-MRS-measured inorganic phosphate/exchangeable phosphate pool (epp) $(p=0.01)$ and reduced phosphocreatine/epp $(p=0.003)$ were observed in the severe injury group indicating persistent cerebral energy depletion. Based on these results, the bNIRS measurement of the oxCCO recovery fraction offers a noninvasive real-time biomarker of brain injury severity within 30 min following $\mathrm{HI}$ insult. (c) The Authors. Published by SPIE under a Creative Commons Attribution 4.0 Unported License. Distribution or reproduction of this work in whole or in part requires full attribution of the original publication, including its DOI. [DOI: 10.1117/1.NPh.6.4.045009]
\end{abstract}

Keywords: broadband near-infrared spectroscopy; near-infrared spectroscopy; cytochrome-c-oxidase; hypoxia-ischemia; neonatal encephalopathy.

Paper 19069R received Jul. 12, 2019; accepted for publication Oct. 14, 2019; published online Nov. 14, 2019.

\section{Introduction}

Neonatal hypoxia-ischemia (HI) is primarily caused by systemic hypoxemia and reduced cerebral blood flow to the brain. Intrapartum-related $\mathrm{HI}$ insults are the third leading cause of death across the world in children under the age of 5. ${ }^{1,2}$ As one of the most common causes of mortality and morbidity in neonates, HI is responsible for nearly one-quarter of all neonatal deaths worldwide. ${ }^{3}$ Neonatal hypoxic-ischemic encephalopathy (HIE) ultimately leads to significant disorders such as cerebral palsy, mental retardation, learning difficulties, and other disabilities. ${ }^{4}$ Preclinical studies of perinatal HI, using newborn pigs (similar gross anatomical features of human neonatal brain $^{5}$ ) have been used to investigate cerebral energetics and

*Address all correspondence to Ilias Tachtsidis, E-mail: i.tachtsidis@ucl .ac.uk metabolism during and after $\mathrm{HI}$ and to develop neuroprotective therapeutic strategies. ${ }^{1,6-10}$

Phosphorous $\left({ }^{31} \mathrm{P}\right)$ and proton $\left({ }^{1} \mathrm{H}\right)$ magnetic resonance spectroscopy (MRS) are gold standard magnetic resonance (MR) techniques for assessing injury severity, as the decline in cerebral energy measured in this manner correlates strongly with neurodevelopmental outcome. ${ }^{11,12}$ Studies have demonstrated that ${ }^{31} \mathrm{P}$-MRS-measured inorganic phosphate/exchangeable phosphate pool ( $\mathrm{Pi} / \mathrm{epp})$, phosphocreatine $(\mathrm{PCr}) / \mathrm{Pi}$, and total nucleotide triphosphate (NTP)/epp $\sim 1$ to $2 \mathrm{~h}$ after HI correlate linearly with the decline in cerebral energetics leading to secondary energy failure. ${ }^{6,10}$ Furthermore, ${ }^{1} \mathrm{H}-\mathrm{MRS}$ lactate/ $\mathrm{N}$-acetylaspartate (Lac/NAA) peak area ratio is the most accurate MR marker of neurodevelopmental outcome in neonates following HIE. ${ }^{13-15}$

MR units are expensive dedicated equipment, run by a specialized team and not always available for in-vivo monitoring of brain metabolism; there are often major issues with using 
${ }^{31} \mathrm{P}-\mathrm{MRS}$ for the induction and monitoring of $\mathrm{HI}$ in the newborn piglet model for neuroprotection. ${ }^{10}$ They are not portable, so patients and/or animals need to be transferred to the magnet room, which carries some risks, especially following an invasive surgery.

Optical technologies such as broadband near-infrared spectroscopy (bNIRS) can provide a noninvasive technique to assess brain tissue oxygenation and metabolism. The bNIRS does this through quantification of changes in concentration of oxyhemoglobin $\left(\left[\mathrm{HbO}_{2}\right]\right)$ and deoxyhemoglobin $([\mathrm{HHb}])$, and oxidation changes of cytochrome-c-oxidase $(\Delta[\mathrm{oxCCO}])$, a unique marker for oxygen metabolism at the cellular level. However, resolving the oxCCO signal in vivo is challenging due to its smaller concentration compared with hemoglobin. ${ }^{16,17}$ Use of the full nearinfrared (NIR) spectrum from 780 to $900 \mathrm{~nm}$ obtained by bNIRS has enabled precise measurement of tissue changes in [oxCCO] in animals, neonates, and adults. ${ }^{18-30}$

Cooper et al. ${ }^{31}$ described the integration of an in-house built bNIRS system with ${ }^{31} \mathrm{P}-\mathrm{MRS}$ to investigate $\mathrm{HI}$ in newborn pigs and discussed the physiological/biochemical relationships of these measurements. They reported that the measurement of $\Delta[$ oxCCO $]$ correlates with changes in energy metabolism measured by ${ }^{31} \mathrm{P}$-MRS during $\mathrm{HI}$ and early recovery after reperfusion and oxygenation. ${ }^{31,32}$ We also demonstrated in our recent study by Bainbridge, et al. ${ }^{10}$ (in 24 piglets measured simultaneously with ${ }^{31} \mathrm{P}-\mathrm{MRS}$ and bNIRS) that ${ }^{31} \mathrm{P}-\mathrm{MRS}$ recovery of energetics were correlated with bNIRS $\Delta[$ oxCCO] recovery within $1 \mathrm{~h}$ following HI. Owing to the highly scattering nature of the brain, hence high attenuation of the incident light, maximizing light collection at tissue interface is crucial in bNIRS to improve measurement accuracy. Therefore, bNIRS systems are developed in-house and utilize broadband light sources and highly sensitive spectrograph detection units. At University College London (UCL), we have been building such instruments since the early 1990s, including (1) UCL1, a single channel bNIRS system; ${ }^{32}$ (2) PHOS, a hybrid optical spectrometer that offers multidistance, multichannel broadband and frequency-domain NIRS systems integration; ${ }^{22}$ (3) cytochrome research instrument and application (CYRIL), which offers multidistance measurements for use in newborns; ${ }^{33}$ (4) and recently a multichannel, multidistance system with imaging capabilities. ${ }^{28}$ UCL-developed bNIRS systems have been used in preclinical models (piglets), in neonates in the intensive care, in healthy adult volunteers, as well as in adult traumatic brain injury patients in the neurointensive care. ${ }^{20,25,30-34}$

These bNIRS systems are bulky, include large spectrographs, with some of them incorporating lens-based systems and large highly sensitive cooled CCDs with large active area and/or high quantum efficiency that are essential for low light levels spectroscopy. On the other hand, recent miniature spectrometers with integrated CCDs offer some favorable characteristics, such as low cost, high sensitivity, and wide availability and offer a solution toward developing compact clinical bNIRS systems. However, the narrow slit (required for high spectral resolution), which is designed with small height in these miniature spectrometers ( $\sim 5$ - to $200 \mu \mathrm{m}$ width $\times 1$ (standard) to $2 \mathrm{~mm}$ height), is almost an order of magnitude shorter than the slit in conventional bNIRS systems, which is about $12 \mathrm{~mm} .{ }^{33}$ This in combination with the high $f$-number $(F / \#$, the ratio of the spectrograph's focal length to the aperture diameter, which is typically $4(F / 4)$ in miniature spectrometers), to produce low stray light inside the spectrometer, greatly limit their light-gathering power or light throughput, which is defined as $\varphi_{c} \propto 1 /(F / \#)^{2}$. This is in direct contrast with the large core and high numerical aperture (NA) of standard optical fibers used in bNIRS systems to collect maximum reflected light from the tissue surface. Furthermore, simulations and in-vivo studies have shown that oxCCO is more precisely measured at larger source-detector separation (SDS) due to the abundance of mitochondria in the cortex compared to extracerebral tissues. ${ }^{26,35,36}$ That is why highly sensitive detection units (large throughput spectrographs with high quantum efficiency CCD cameras) are required to accurately measure small light intensities ${ }^{37}$ since light is strongly attenuated before reaching the detector at larger SDS (attenuation of $10^{3}$ to $10^{4}$ at $30 \mathrm{~mm} \mathrm{SDS}^{38,39}$ ).

Some researchers have tackled the limitations of miniature spectrometers (in particular QE65000 from Ocean Optics, Inc.) by removing the narrow entrance slit of the spectrometer and using the detector fiber $(\mathrm{NA}=0.22$ ) to act as the entrance slit while modifying its design to match the form factor of the original slit of the miniature spectrometer in order to maintain the optical resolution. ${ }^{40-42}$ According to our investigations, high NA fibers and low $f$-number spectrometers are essential components of a bNIRS system for measuring [oxCCO]. We observed that using a modified geometry fiber to couple light into the QE65 Pro enhances the transmission intensity across 780 to $900 \mathrm{~nm}$ by $303 \pm 56 \%$ with almost fourfold increase in the peak intensity at $810 \mathrm{~nm}$, compared to when a larger NA $(0.57)$ standard fiber is used with the same spectrometer. We further demonstrated that using a larger light throughput miniature spectrometer (lower $f$-number) combined with a standard NA $=0.57$ optical fiber further increased the transmission intensity across 780 to $900 \mathrm{~nm}$ by $665 \pm 146 \%$ (and sevenfold increase in peak intensity at $810 \mathrm{~nm}$ ), compared to when the same optical fiber coupled the collected light into the highly sensitive QE65 Pro. ${ }^{43}$

The aim of this study is to present the development of a miniature bNIRS system called miniCYRIL for measuring brain tissue change in $\left[\mathrm{HbO}_{2}\right],[\mathrm{HHb}]$, and [oxCCO]. This is based on a miniature light source and a customized large light throughput miniature spectrometer coupled to high NA optical fibers. In addition, we describe the utilization of miniCYRIL in a preclinical study of perinatal HI. We report how the miniCYRIL measurements of brain tissue hemoglobin oxygenation and oxCCO can be used to predict the severity of primary brain injury in piglets following $\mathrm{HI}$ as prognosticated by cerebral Lac/NAA measurements of ${ }^{1} \mathrm{H}-\mathrm{MRS}, 24 \mathrm{~h}$ post HI. Our aim is to determine whether bNIRS can distinguish injury severity during the first half-hour after $\mathrm{HI}$ as this might have significant impact on our understanding of injury severity and a basis for further decision-making regarding the application of appropriate neuroprotection strategy in the preclinical model.

\section{Methods}

\section{1 miniCYRIL Instrumentation}

\subsubsection{Hardware}

The miniCYRIL device is a single-channel bNIRS system and uses a thermally stabilized miniature white-light source (HL2000-HP)—a $20 \mathrm{~W}$ tungsten halogen lamp (Ocean Optics). The light source is compact and small $(6.2 \times 6 \times 15 \mathrm{~cm})$ and produces a continuous spectral output in the NIR region.

The source and detector optical fibers are identical bundles and custom-built by Loptek (Germany) with high NA (NA = $0.57,2 \theta c \sim 70 \mathrm{deg}, F / 0.1$ ) for maximum illumination and light 
collection. The fibers were made with a 90-deg bend at the subject end to ensure efficient light collection from the tissue surface. The core diameter is $2.3 \mathrm{~mm}$ to match the subminiature version A connector and both fibers are magnet-compatible and 7-m long to enable simultaneous MRS measurements when needed. Optodes were placed colinear across the head as shown in Fig. 1, and the measurements were performed in transmission mode through the piglet's head with SDS of 40 to $45 \mathrm{~mm}$ depending on the piglet's head size.

The detection unit of miniCYRIL is a customized version of the Ventana VIS-NIR miniature spectrometer (Ocean Optics). The large throughput design (low $f$-number, $F / 2$ ) of the Ventana spectrometer is advantageous over the other Ocean Optics QE-spectrometers, which have been used in other studies, ${ }^{40-42,44}$ as it allows more of the collected light into the spectrometer (since the light collection capability of all spectrometers is inversely proportional to the $f$-number squared). Furthermore, the use of a volume phase holographic grating instead of ruled grating provides maximum diffraction efficiency with little stray light inside the spectrometer.

The main challenge with the miniaturization of the bNIRS systems arises from the intrinsic contrast between the high NA of the optical fibers and the small throughput design of the spectrometers to maintain a flattened spectral field. Despite their wide availability, low cost, and compact nature, a limitation of the off-the-shelf spectrometers is that the optical bench design of the miniature spectrometers cannot be customized for bNIRS. Therefore, we took other approaches to enhance the throughput of QE65 Pro, the upgrade version of QE65000 that has been used in other studies ${ }^{45-47}$ due to its highly sensitive CCD with thermoelectric cooling at $-15^{\circ} \mathrm{C}$. We attempted to match the $f$-numbers of the optical fiber to the spectrometer using external collimating and focusing lenses, which enhanced the light throughput of the QE65 Pro by three times. However, the Ventana VIS-NIR, with lower $f$-number, increased the light throughput by an order of magnitude. This was achieved despite

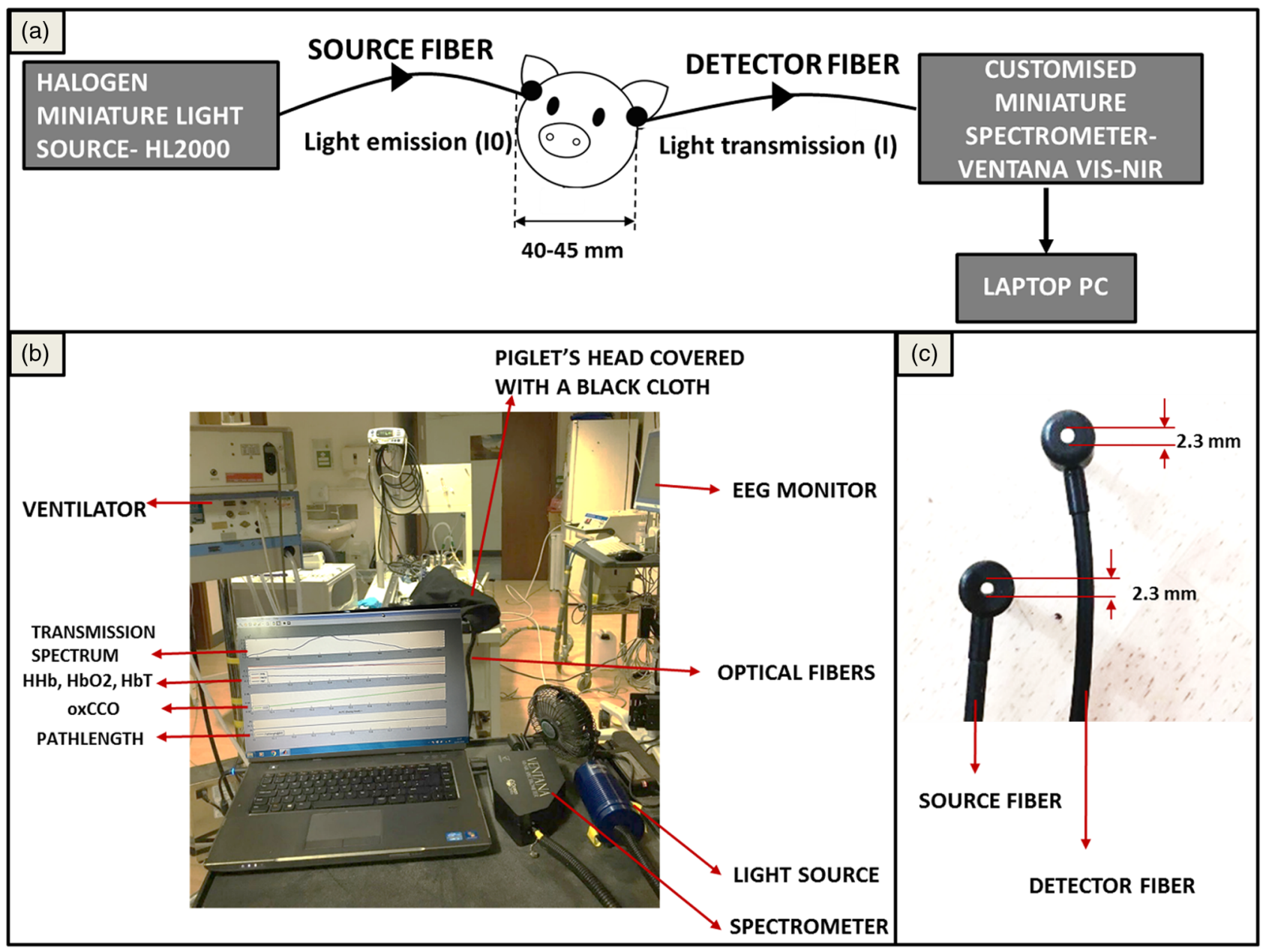

Fig. 1 miniCYRIL setup in a preclinical study of neonatal HIE. (a) A schematic diagram of the experimental design. (b) miniCYRIL's components including a miniature white-light source (HL2000-HP), a customized Ventana VIS-NIR miniature spectrometer, optical fibers, and a laptop for the real-time display of concentration data. Light from the halogen light source is emitted onto the piglet brain $\left(I_{0}\right)$ through a 0.57-NA optical fiber with 2.3-mm diameter (c). Light transmitted through the brain (I) is collected at tissue surface (SDS: 40 to $45 \mathrm{~mm}$ ) through an identical optical fiber and transferred to the miniature spectrometer. A MATLAB program then converts the collected spectral data to changes in attenuation and accordingly calculates changes in the concentration of $\mathrm{HHb}, \mathrm{HbO}_{2}$, and oxCCO based on the measured optical pathlength. The spectral and concentration data are displayed in real time on a laptop screen (b) and saved in a CSV file after each measurement. 
Table 1 Comparison of the miniature spectrometers QE65 Pro and customized Ventana VIS-NIR.

\begin{tabular}{lcc} 
Specifications & QE65 Pro & $\begin{array}{c}\text { Customized } \\
\text { Ventana }\end{array}$ \\
\hline Light throughput & $F / 4$ & $F / 2$ \\
Bandwidth & $\begin{array}{c}353 \mathrm{~nm}(700 \\
\text { to } 1053 \mathrm{~nm})\end{array}$ & $\begin{array}{c}677 \mathrm{~nm}(430 \\
\text { to } 1100 \mathrm{~nm})\end{array}$ \\
Spectral resolution & $3.5 \mathrm{~nm}$ & $4 \mathrm{~nm}$ \\
CCD peak quantum & $95 \%$ & $75 \%$ \\
efficiency & & $14 \mu \mathrm{m}^{2}$ \\
CCD pixel size & $24.6 \mu \mathrm{m}^{2}$ & $1024 \times 64$ \\
CCD pixels & $1024 \times 58$ & $14.336 \times 0.896 \mathrm{~mm}^{2}$ \\
CCD active area & $24.576 \times 1.392 \mathrm{~mm}^{2}$ & $15^{\circ} \mathrm{C}\left(10^{\circ} \mathrm{C}\right.$ \\
CCD temperature & $-15^{\circ} \mathrm{C}\left(40^{\circ} \mathrm{C}\right.$ & below ambient $)$ \\
& below ambient $)$ & 6 counts RMS $^{2}$ \\
$\begin{array}{l}\text { Dark noise } \\
\text { (single spectrum) }\end{array}$ & $3 \mathrm{counts} \mathrm{RMS}$ & \\
SNR & $1000: 1$ & $500: 1$ \\
\hline
\end{tabular}

the Ventana spectrometer having a less sensitive $\mathrm{CCD}$ and smaller signal-to-noise ratio (SNR) compared to the CCD for QE65 Pro (quantum efficiency $75 \%$ versus $95 \%$, active area $\sim 14$ versus $25 \mathrm{~mm}^{2}$, and SNR 500:1 versus 1000:1). ${ }^{43}$ As seen in Table 1, summarizing the spectroscopic and detector specifications of QE65 Pro and customized Ventana spectrometer, one of the most important parameters to consider in adapting a miniature spectrometer for bNIRS application is the light throughput ( $f$-number).

The only drawback of Ventana for bNIRS is the absence of a thermoelectric cooling system for the CCD. This leads to a considerably large dark count, which also varies over time. The stability of dark count is crucial in bNIRS, especially for long measurements (hours), because the differential spectroscopy technique uses the first intensity measurement to calculate the relative attenuation change. In fact, including or subtracting an inconsistent dark spectrum in the measurement algorithm results in a false interpretation of change in the intensity and attenuation of light and consequently causes significant errors in the concentration measurement. Therefore, we retrofitted a cooling system inside the Ventana spectrometer with the help of the manufacturers (Wasatch Photonics) to stabilize the CCD temperature at $\sim 15^{\circ} \mathrm{C}$. The design is limited to cooling at $15^{\circ} \mathrm{C}$ $\left(\sim 10^{\circ} \mathrm{C}\right.$ below ambient) to prevent condensation forming on the sensor at lower temperatures.

\subsubsection{Software}

A MATLAB compatible driver from Ocean Optics was installed on a laptop to control the USB spectrometer and a program was written to collect and process the spectral data from the brain.

Change in attenuation was calculated and interpolated to the nearest nanometer across 780 to $900 \mathrm{~nm}$ using a spline interpolation. The UCLn algorithm [Eq. (1)] was then applied to resolve changes in $[\mathrm{HHb}],\left[\mathrm{HbO}_{2}\right]$, and [oxCCO] across 120 wavelengths using their specific extinction coefficient spectra while accounting for the wavelength dependence of the differential pathlength factor (DPF). ${ }^{17}$ The UCLn is a least-squares regression analysis, which finds the best fit of chromophore concentration change $(\Delta c)$ based on the chromophore extinction coefficients $\left(\varepsilon_{\lambda}\right)$ (Fig. 7, Sec. 7), the measured attenuation changes over $n$ wavelengths $\left(\Delta A_{\lambda}\right)$ and the optical pathlength of light through the tissue. ${ }^{16}$

$\left[\begin{array}{c}\Delta c_{\mathrm{HHb}} \\ \Delta c_{\mathrm{HbO} 2} \\ \Delta c_{\mathrm{oxCCO}}\end{array}\right]=\frac{1}{\text { Pathlength }(\lambda)} \times\left[\begin{array}{ccc}\varepsilon_{\mathrm{HHb}, \lambda 1} & \varepsilon_{\mathrm{HbO} 2, \lambda 1} & \varepsilon_{\mathrm{oxCCO}, \lambda 1} \\ \varepsilon_{\mathrm{HHb}, \lambda 2} & \varepsilon_{\mathrm{HbO} 2, \lambda 2} & \varepsilon_{\mathrm{oxCCO}, \lambda 2} \\ \vdots & \vdots & \vdots \\ \varepsilon_{\mathrm{HHb}, \lambda n} & \varepsilon_{\mathrm{HbO} 2, \lambda n} & \varepsilon_{\mathrm{oxCCO}, \lambda n}\end{array}\right]^{-1} \times\left[\begin{array}{c}\Delta A_{\lambda 1} \\ \Delta A_{\lambda 2} \\ \vdots \\ \Delta A_{\lambda n}\end{array}\right]$.

The optical pathlength through the tissue in existing bNIRS systems is calculated via multiplying the SDS by DPF, a scaling factor that is typically measured by time-domain ${ }^{48,49}$ and frequency-domain NIRS systems. ${ }^{50,51}$

In this study, we estimated the optical pathlength in real time [Eq. (2)]. We first acquired a reference spectrum to characterize the input light and the pathlength for the 840 -nm water absorption feature was obtained by fitting the second differential of the attenuation spectra to the second differential of in-vitro water signal between 800 and $880 \mathrm{~nm}$ and assuming $85 \%$ water content. 52

Pathlength $(840 \mathrm{~nm})=\frac{\left[\varepsilon_{H 2 O_{t}}(\lambda)^{\prime \prime}\right]^{-1} \cdot \mathrm{A}(\lambda)^{\prime \prime}}{0.85}$.

The algorithm was corrected for the wavelength dependence of pathlength, according to the work done by Essenpreis et al. ${ }^{53}$ in 1993, to account for decreasing scattering effects at increasing wavelengths in tissue. Then we calculated changes $(\Delta)$ in the brain concentration of $\mathrm{HHb}, \mathrm{HbO}_{2}$, and oxCCO in real time. Accordingly, changes in hemoglobin difference $(\Delta[\mathrm{HbDiff}]=$ $\Delta\left[\mathrm{HbO}_{2}\right]-\Delta[\mathrm{HHb}]$; indicating changes in brain oxygenation) and total hemoglobin $\left(\Delta[\mathrm{HbT}]=\Delta\left[\mathrm{HbO}_{2}\right]+\Delta[\mathrm{HHb}]\right.$; indicating cerebral blood volume) were also estimated.

\subsection{3 miniCYRIL device characterization}

Dark noise. The modified Ventana spectrometer was characterized for its dark count and noise performance before and after customization (the retrofit cooling system). To investigate the thermal noise and the dark count of the CCD detector in terms of magnitude and stability over time since several hours of bNIRS measurement is required for the study of $\mathrm{HI}$ in piglets, intensity data were acquired for $10 \mathrm{~h}$ with the slit of the spectrometer closed (CCD integration time set at $10 \mathrm{~s}$ ). Dark count 
distribution between 780 and $900 \mathrm{~nm}$ and the change in the intensity of individual wavelengths over time were investigated.

Stability. To test the stability of the system over time, studies on solid phantoms with tissue-like optical properties $\left[\mu_{a}=\right.$ 0.02 and $\left.\mu_{s}{ }^{\prime}=0.9\left(\mathrm{~mm}^{-1} \mathrm{~mol}^{-1}\right)\right]$ were performed using miniCYRIL. Changes in the spectral data and chromophore concentrations on the phantom were measured during dark (no light through the phantom) and when light from the light source was incident on it at an SDS of $4 \mathrm{~cm}$.

\subsection{Preclinical Study}

\subsubsection{Animal preparation}

All animal experiments were approved by the UCL Ethics Committee and performed under the UK Home Office Guidelines [Animals (Scientific Procedures) Act, 1986]. A total of 27 term-born piglets, aged less than $36 \mathrm{~h}$ were anesthetized and surgically prepared, as previously described. ${ }^{1,10,54}$ Briefly, animals were sedated with intramuscular midazolam $(0.2 \mathrm{mg} /$ $\mathrm{kg})$ and anesthetized with isoflurane mixed with air $(3 \% \mathrm{v} / \mathrm{v}$ during surgery, $1.5 \%$ to $2.5 \%$ during experimentation) to remain unconscious throughout the experiment. The animals were mechanically ventilated through tracheostomy (SLE 2000 infant ventilator, Surrey, UK) and arterial oxygen saturation $\left(\mathrm{SpO}_{2}\right)$ was monitored (Nonin Medical) continuously. The common carotid arteries were surgically isolated and carefully surrounded by remotely inflatable vascular occluders (OC2A, InVivo Metric). An umbilical arterial line was inserted for invasive mean arterial blood pressure (MABP) and heart rate (HR) monitoring, and an umbilical venous line was inserted for infusion administration. A multichannel EEG (Nicolet) was used to record the electrical activity of the piglet brain during baseline, $\mathrm{HI}$ insult, and continuously for $48 \mathrm{~h}$ after $\mathrm{HI}$.

\subsection{2 miniCYRIL measurements}

The experimental setup for bNIRS measurement is presented in Fig. 1. Prior to the experiment, a reference spectrum was acquired for $30 \mathrm{~s}$ using a poster tube to record the intensity of the light source through free space with no absorbing medium while avoiding ambient light interference (CCD integration time $=50 \mathrm{~ms}$ ). The intensity spectra were saved as a MATLAB file to be processed for real-time calculation of the optical pathlength.

Animals were positioned prone in a plastic pod with the head immobilized in a stereotactic frame. The pod was equipped with the miniCYRIL probe holders against the sides of the head and built within a purpose-built MR compatible transport incubator.

The source and detector fibers were securely fitted in the probe holders, which were provided with tightening screws to make sure good contact with the scalp is achieved. The diffused light was collected from the surface of the head using the detector fiber at a sampling rate of $0.1 \mathrm{~Hz}$ (CCD integration time $=10 \mathrm{~s}$ ) and were displayed along with the calculated concentration data on a laptop.

\subsection{3 ${ }^{31} \mathrm{P}$ and ${ }^{1} \mathrm{H}$ magnetic resonance spectroscopy}

MRS was performed at 24 and $48 \mathrm{~h}$ post $\mathrm{HI}$ in a Philips clinical 3T MRI scanner. Whole-brain ${ }^{31} \mathrm{P}$-MRS spectra were acquired with 1-min resolution and analyzed using the Advanced Method for Accurate, Robust, and Efficient Spectral fitting of MRS data $^{55}$ as implemented in the jMRUI software. NTP is predominantly composed of adenosine triphosphate (ATP); thus, changes in this signal during the experiments reflected changes in ATP. Measurements of $\mathrm{Pi}, \mathrm{PCr}$, epp $(\mathrm{epp}=\mathrm{Pi}+\mathrm{PCr}+2 \gamma$ $\mathrm{NTP}+\beta$-NTP) were acquired over the whole brain, and peak area ratios were calculated (Pi/epp, PCr/epp, and NTP/epp). ${ }^{10}$

Brain $\mathrm{pH}$ was estimated using the chemical shift separation of Pi with $\mathrm{PCr}^{56,57}$ The Pi signal was fitted using three singlet components, and $\mathrm{pH}$ was estimated using the amplitudeweighted mean chemical shift separation.

${ }^{1} \mathrm{H}$-MRS spectra were also acquired, measuring metabolites in a white matter voxel in the dorsal right subcortical region $(8 \times 8 \times 15 \mathrm{~mm})$ and deep gray matter voxel $(15 \times 15 \times 10 \mathrm{~mm})$ in the thalamus. The data were analyzed using TARQUIN software; we included threonine in the lactate fit and the Lac/NAA peak area ratio was calculated. The inclusion of threonine with lactate measurement has shown to contribute better prediction of outcome in neonatal HIE after therapeutic hypothermia. ${ }^{15}$

\subsubsection{Hypoxia-ischemia protocol}

Animals were monitored for $1 \mathrm{~h}$ to ensure hemodynamic stability, normal EEG, and stable miniCYRIL baseline were established prior to the $\mathrm{HI}$ insult. At the start of $\mathrm{HI}$, the carotid occluders were inflated and fraction of inspired oxygen $\left(\mathrm{FiO}_{2}\right)$ was simultaneously reduced to $6 \%$. Reduction of $\mathrm{FiO}_{2}$ to $6 \%$ took about 3 min after which the $\mathrm{FiO}_{2}$ was held at this value for around 20 to 22 minutes (duration of $\mathrm{HI}$ ) while monitoring the drop in the oxCCO signal $(\Delta[\mathrm{oxCCO}])$ with the miniCYRIL system (at this stage, the electrical activity of the brain was suppressed, and the EEG was flat). If during HI the oxCCO signal dropped below $-3.5 \mu \mathrm{M}$ from the baseline or the MABP reduced below $27 \mathrm{mmHg}$, the $\mathrm{FiO}_{2}$ was titrated (1\% every minute) to stabilize the animal. Blood gas analysis was performed every 5 min during HI. At the end of the insult period, the occluders were deflated and oxygen $\left(\mathrm{FiO}_{2}\right)$ was returned to room air while the animal was continuously monitored with bNIRS, EEG, and physiological data for $48 \mathrm{~h}$. The total HI period was decided by two experienced team members based on the duration of isoelectric $\mathrm{EEG}$, hypotension (MABP $<30 \mathrm{mmHg}$ ), area under the curve (AUC) of the oxCCO signal as measured by miniCYRIL, total reduction in $\mathrm{FiO}_{2}\left(\mathrm{AUC}-\mathrm{FiO}{ }_{2}\right)$, and blood gases.

All animals received therapeutic hypothermia (whole body cooling at $\left.33.5^{\circ} \mathrm{C}\right) 1 \mathrm{~h}$ after $\mathrm{HI}$, with some receiving additional treatment interventions. Moreover, some animals had pharmacological interventions to sustain systemic physiological normality while being under continuous physiological monitoring and intensive life support throughout the 48-h experiment.

\subsection{Data Analysis}

Data analysis was carried out using MATLAB 2015 (MathWorks) and XLSTAT 2018.

\subsubsection{Optical data quality control}

To investigate the spectral changes accounted to oxCCO, the UCLn algorithm was used to derive changes in chromophore concentrations at the nadir of $\mathrm{HI}$ when solving only for two chromophores $\left(\mathrm{HHb}\right.$ and $\left.\mathrm{HbO}_{2}\right)$ as well as when solving for all three chromophores $\left(\mathrm{HHb}, \mathrm{HbO}_{2}\right.$, and oxCCO). ${ }^{32}$ The attenuation change spectra were then back-calculated from the concentration changes at the nadir of $\mathrm{HI}$ and the difference 
between the measured attenuation spectra with the two- and three-chromophore fit were studied. If all the chromophores that determine the attenuation spectra were fitted, then the residual error from the measured attenuation and back-calculated attenuations must be almost zero having no defined shape. However, if the residuals have defined shapes, it would suggest that there was a chromophore that is unaccounted for in the calculations. This analysis was carried out using a MATLAB script for all the experiments.

\subsubsection{Recovery fraction}

An algorithm was developed to quantify the recovery of bNIRS measurements of $\Delta[\mathrm{HbT}], \Delta[\mathrm{HbDiff}]$, and $\Delta[\mathrm{oxCCO}]$ after HI. The bNIRS signals were closely monitored for a stable period following HI, which occurred during the first $30 \mathrm{~min}$ after the resuscitation. Data at baseline and recovery were averaged over a 1-min window to calculate the recovery fraction of each signal relative to baseline. All the measurements were normalized against the nadir point as seen in Eq. (3).

Recovery Fraction $(\mathrm{RF})=\frac{\text { Recovery after HI }- \text { Nadir }}{\text { Baseline }- \text { Nadir }} \times 100 \%$.

\subsubsection{Receiver operating characteristic}

The receiver operating characteristic (ROC) analysis was performed in an iterative approach to select an optimal cut-off value of a potential prognostic marker for clinical use. ROC curves compare the sensitivity (percentage of true positives) against the specificity (percentage of true negatives) across a range of values to predict a dichotomous outcome. In this study, ROC analysis was carried out in XLSTAT to investigate how the recovery fraction of bNIRS signals ( $\Delta[\mathrm{HbT}], \Delta[\mathrm{HbDiff}]$, and $\Delta[\mathrm{oxCCO}])$ can be used to prognosticate the severity of brain injury, as measured by the ${ }^{1} \mathrm{H}$-MRS-measured Lac/NAA at $24 \mathrm{~h}$. The ROC-AUC represents how well the recovery fraction of the bNIRS signals are able to distinguish between the two diagnostic groups (mild and severe brain injuries). ${ }^{58}$

In this study, sensitivity and specificity were used to determine the ability of the recovery fraction of oxCCO to detect a severe injury following HI. Sensitivity refers to the proportion of animals who were diagnosed with severe brain injuries at 24 h post HI, having thalamic Lac/NAA of $\geq 0.39$, and specificity is the proportion of the animals with mild brain injuries (Lac/NAA < 0.39). Thalamic Lac/NAA cut-off value of 0.39 indicates clinical outcome in the neonatal population after hypothermia following neonatal hypoxic-ischemic encephalopathy, ${ }^{15}$ and it is used as a clinical biomarker for prognostication. Plotting the sensitivity and specificity against the recovery fractions of bNIRS signals provides a range of cut-off points with different sensitivities and specificities. The bNIRS measurement with the highest ROC-AUC and a cut-off value with the highest sensitivity and specificity has the highest potential to be used as a prognostic measure to separate between mild and severe injury groups.

\subsubsection{Statistics}

Statistical analysis was nonparametric and was carried out in XLSTAT, Microsoft Excel. The Mann-Whitney test was performed to compare the recovery fraction of the bNIRS measurements $(\Delta[\mathrm{HbT}], \Delta[\mathrm{HbDiff}]$, and $\Delta[\mathrm{oxCCO}])$ between two groups of mild and severe injuries, separated based on the optimal cut-off point for the bNIRS signal with highest ROC-AUC and highest sensitivity and specificity. Other data that were compared this way included 1-min average of HR and MABP within $30 \mathrm{~min}$ of the recovery period, as well as the absolute ratios of ${ }^{1} \mathrm{H}-\mathrm{MRS}$ [Lac/NAA (thalamus) and Lac/NAA (white matter)] and ${ }^{31} \mathrm{P}-\mathrm{MRS}$ (PCr/epp, Pi/epp, NTP/epp, and pH) at 24 and $48 \mathrm{~h}$ post HI. When group data were used, results were presented as median \pm interquartile range (IQR).

\section{Results}

\section{1 miniCYRIL Device Characterization}

The dark count of the original Ventana spectrometer was $8850 \pm$ 725 counts (for 10-s integration time) across all wavelengths, which was increased by $35 \%$ after $10 \mathrm{~h}$. After retrofitting the cooling system in the modified Ventana, the dark count was reduced by $75 \%(2440 \pm 218)$ and it was stable over $10 \mathrm{~h}$ with less than $1 \%$ increase.

Phantom measurements showed that the performance of miniCYRIL (with the customized Ventana spectrometer) is stable over long measurement hours. The average change in the intensity of light traversing the phantom was less than $8 \%$ during 14-h measurement (part of it is due to the optical drift of the light source, which is $<0.3 \%$ per hour ${ }^{59}$ ). The concentration signals were flat with no prominent features $[\Delta[\mathrm{HHb}]=0.59 \pm 0.19$ $(\mu \mathrm{M} \times \mathrm{cm}), \Delta\left[\mathrm{HbO}_{2}\right]=0.02 \pm 0.08(\mu \mathrm{M} \times \mathrm{cm}), \Delta[\mathrm{oxCCO}]=$ $-0.30 \pm 0.09(\mu \mathrm{M} \times \mathrm{cm})]$ (see Sec. 6).

\section{2 miniCYRIL Measurements during Hypoxia-Ischemia}

miniCYRIL measurements were recorded in 27 piglets during baseline, HI, and $30 \mathrm{~min}$ of the recovery period after the HI insult. An example of changes in the intensity of light traversing the piglet's brain during baseline $\left(\mathrm{I}_{1}\right)$ and $\mathrm{HI}\left(\mathrm{I}_{2}\right)$ is shown in Fig. 2(a). The average peak and mean intensity light emission and transmission across the bandwidth of interest (780 to $900 \mathrm{~nm}$ ) in all piglets are presented in Table 2. The intensity spectra recorded by miniCYRIL have two features around 760 and $830 \mathrm{~nm}$, which are attributed to $\mathrm{HHb}$ and oxCCO. During HI, a larger number of photons pass through the brain due to the reduction in cerebral blood volume, and hence less attenuation.

Changes in the attenuation during baseline $(\Delta \mathrm{A} 1)$ and at the nadir of $\mathrm{HI}(\Delta \mathrm{A} 2)$, relative to the first measurement, are presented in Fig. 2(b). At baseline, there is no major change in the magnitude and the shape of the attenuation spectrum, relative to the first measurement. However, during HI, there is a significant change in the magnitude and shape of the attenuation spectrum due to the physiological changes in the brain, i.e., reduction in blood flow and oxygenation.

Changes in the concentration of $\mathrm{HHb}, \mathrm{HbO}_{2}$, and oxCCO were accordingly calculated in real time from the measured change in attenuation, as explained in Sec. 2.1.2. Figures 2(c)-2(e) illustrate changes in the systemic data as well as the bNIRS measurements in one piglet during HI.

At baseline when the piglet was in normal physiological condition $\left(\mathrm{SpO}_{2}=98 \%, \mathrm{HR}=235 \mathrm{bpm}, \quad\right.$ and $\mathrm{MABP}=$ $50 \mathrm{mmHg}$ ) and there were no variations in oxygen levels 


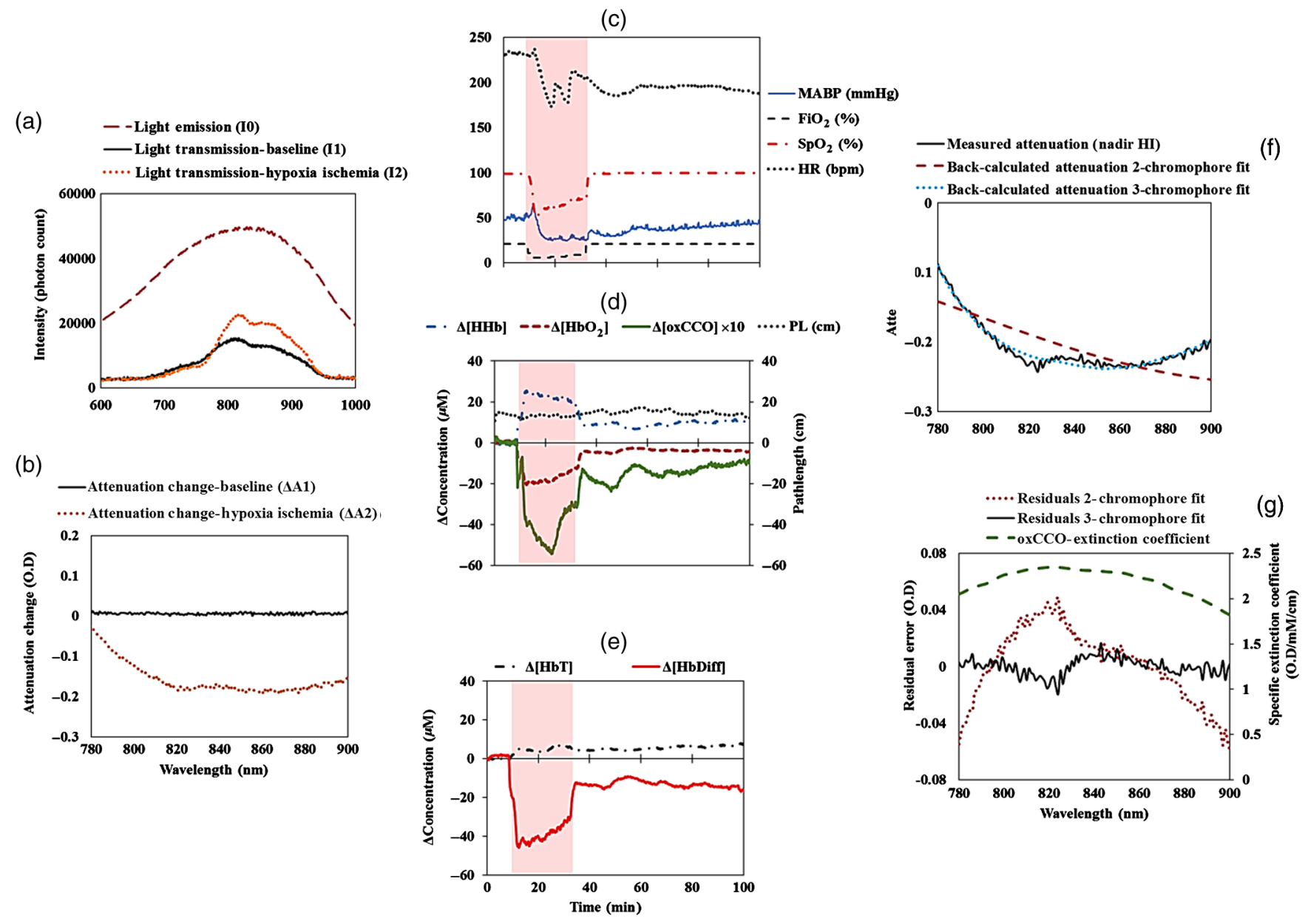

Fig. 2 An example of the optical data from a piglet (LWP483). (a) Incident $\left(\mathrm{I}_{0}\right)$ and transmitted light spectra $\left(\mathrm{I}_{1}\right.$ and $\left.\mathrm{I}_{2}\right)$ through the brain during baseline and $\mathrm{HI}$. The significantly reduced photon count in $\mathrm{I}_{1}$ and $\mathrm{I}_{2}$ is due to the optical properties of the piglet brain. The increased peak count in $I_{2}$ relative to $I_{1}$ is due to less absorption during $\mathrm{HI}$. (b) Attenuation spectra measured at baseline and insult. There is no major change in the attenuation spectrum relative to the first measurement at baseline $(\Delta \mathrm{A} 1)$. However, during $\mathrm{HI}$, there is a significant change in the magnitude and the shape of the attenuation spectrum due to the physiological changes in the brain, i.e., reduction in blood flow and oxygenation ( $\Delta \mathrm{A} 2)$. (c) Changes in the systemic physiological data at baseline, $\mathrm{HI}$, and recovery. (d) and (e) Changes in the bNIRS measurements baseline, $\mathrm{HI}$, and the recovery period as measured by miniCYRIL based on second derivative spectroscopy for pathlength change measurement. The shaded area indicates the HI period. There is no change in the optical pathlength during $\mathrm{HI}$ and recovery. (f) Measured attenuation spectrum at $\mathrm{HI}$ and back-calculated attenuations from two- and three-chromophore fit. (g) Residual errors from twoand three-chromophore fit. The two-chromophore fit residual is similar to the extinction coefficient of oxCCO, whereas the three-chromophore fit residual error is approximately zero.

$\left(\mathrm{FiO}_{2} \sim 21 \%\right)$, all the miniCYRIL signals were stable. As soon as HI was initiated (shown in the shaded red area), all the systemic data including $\mathrm{SpO}_{2}, \mathrm{MABP}$, and HR dropped [Fig. 2(c)] and simultaneously, bNIRS measurement of $\Delta\left[\mathrm{HbO}_{2}\right]$ declined and $\Delta[\mathrm{HHb}]$ increased [Fig. 2(d)], resulting in a significantly large drop in $\Delta[$ HbDiff $]$ due to the limited oxygen availability (hypoxia) in the brain [Fig. 2(e)]. Blood volume as measured by $\Delta[\mathrm{HbT}]$, slightly increased at the start of $\mathrm{HI}$ and remained stable during $\mathrm{HI}$ due to the restriction of blood flow, caused by the occlusion of both carotid arteries [Fig. 2(e)]. Measured $\Delta[\mathrm{oxCCO}]$ also declined significantly because of the insufficient blood supply and limited oxygen availability [Fig. 2(d)]. In other words, during HI, oxygen as the final electron acceptor in the mitochondrial electron transport chain became significantly reduced, which consequently led to inhibition of cytochrome- c-oxidase $(\mathrm{CCO})$ oxidization until it reached a nadir value before $\mathrm{FiO}_{2}$ was titrated or restored to $21 \%$ at the end of $\mathrm{HI}$. At the end of insult, when occluders were deflated and oxygen was revived (see the $\mathrm{SpO}_{2}$ measurement), all the physiological and miniCYRIL measurements started recovering back toward the baseline values [Figs. 2(c)-2(e)].

Figure 2(f) shows the measured attenuation spectrum at the nadir of $\mathrm{HI}$ (minimum $\Delta[\mathrm{oxCCO}]$ ), as well as the backcalculated attenuations from two- and three-chromophore fit. When solving only for two chromophores, all the chromophores were not accounted for as the back-calculated attenuation from the the two-chromophore fit did not match the measured attenuation. Accordingly, the residual error from the two-chromophore fit, as shown in Fig. 2(g), had a well-defined shape that matched the extinction coefficient spectrum of oxidized-reduced $\mathrm{CCO}$ 
Table 2 Average peak intensity of light emission and transmission through the piglet brain measured with miniCYRIL at baseline (SDS: 40 to $45 \mathrm{~mm}$ ) in 27 piglets.

\begin{tabular}{|c|c|c|}
\hline & $\begin{array}{c}\text { Photon count } \\
\text { (mean } \pm \text { standard } \\
\text { deviation)/ } \\
\text { integration time }\end{array}$ & $\begin{array}{l}\text { Wavelength } \\
\quad(\mathrm{nm})\end{array}$ \\
\hline \multicolumn{3}{|l|}{ Light emission $\left(I_{0}\right)$} \\
\hline $\begin{array}{l}\text { Group mean peak intensity } \\
\text { at a single wavelength }\end{array}$ & $52981 \pm 54 / 100 \mathrm{~ms}$ & $839 \pm 6$ \\
\hline $\begin{array}{l}\text { Group mean intensity across } \\
\text { all wavelengths }\end{array}$ & $50817 \pm 9 / 100 \mathrm{~ms}$ & 780 to 900 \\
\hline \multicolumn{3}{|l|}{ Light transmission (I) } \\
\hline $\begin{array}{l}\text { Group mean peak intensity } \\
\text { at a single wavelength }\end{array}$ & $12625 \pm 4662 / 10 \mathrm{~s}$ & $811 \pm 4$ \\
\hline $\begin{array}{l}\text { Group mean intensity across } \\
\text { all wavelengths }\end{array}$ & $10463 \pm 3782 / 10 \mathrm{~s}$ & 780 to 900 \\
\hline
\end{tabular}

between 780 and $900 \mathrm{~nm}$ with a peak around $830 \mathrm{~nm}$ (Fig. 7, Sec. 7). This suggests that resolving only for $[\mathrm{HHb}]$ and $\left[\mathrm{HbO}_{2}\right]$ would leave a chromophore with the spectral features of oxCCO unaccounted for. In contrast, the residual spectrum from the three-chromophore fit was negligible with no defined shape, compared to the residual from the two-chromophore fit. This suggests that when solving for three chromophores, which has been done in this study, we could genuinely measure [oxCCO] in the field of view and all the main chromophores would be accounted for in the measurement. It should be mentioned that the appearance of a small dip around $820 \mathrm{~nm}$ in the residual signal from the three-chromophore fit [Fig. 2(g)] could be due to increased spectral noise or signals from other unresolved chromophores such as cytochrome-c or cytochrome-b. However, in this study, we are mainly interested in measuring [oxCCO], and the residual analysis demonstrates that our oxCCO measurement is authentic and not simply a cross talk artifact of the larger hemoglobin signals.

\subsection{ROC Analysis: Identifying an Early bNIRS Prognostic Marker for HI Injury Severity in the Preclinical Model of Neuroprotection}

The miniCYRIL recovery fraction signals that were monitored for the ROC analysis include hemoglobin difference (HbDiff, cerebral oxygenation), total hemoglobin (HbT, cerebral blood volume), and cerebral metabolism through the measurement of oxCCO. Of the 27 piglets in this study, 13 had thalamic Lac/NAA of $\geq 0.39$ (having a severe injury) at $24 \mathrm{~h}$ post $\mathrm{HI}$ and 14 piglets had Lac/NAA of $<0.39$ (having a mild injury), as seen in Table 3. The ROC curves for the recovery fractions of $\Delta[\mathrm{oxCCO}], \Delta[\mathrm{HbDiff}]$, and $\Delta[\mathrm{HbT}]$ had AUCs of $0.99,0.90$, and 0.58 , respectively, as shown in Fig. 3(a). This suggests that the recovery fraction of $\Delta[\mathrm{oxCCO}]$ is the best classifier of outcome (mild or severe brain injury). Therefore, piglets were classified into two groups of mild injury and severe injury based on the cut-off recovery fraction of oxCCO $(79 \%)$ : a recovery
Table 3 Recovery fractions of the bNIRS data in 27 piglets within $30 \mathrm{~min}$ post HI. The $24-\mathrm{h} \mathrm{Lac} / \mathrm{NAA}$ of $\geq 0.39$ is used as a cut-off threshold for mild (0) and severe injury (1). ${ }^{15}$

\begin{tabular}{|c|c|c|c|c|c|}
\hline Piglets & $\begin{array}{c}\text { 24-h Lac/NAA } \\
\text { (thalamus) }\end{array}$ & $\begin{array}{l}\text { Outcome } \\
\text { (mild/ } \\
\text { severe) }\end{array}$ & $\begin{array}{c}\Delta[\mathrm{oxCCO}] \\
\text { RF (\%) }\end{array}$ & $\begin{array}{c}\Delta[\mathrm{HbDiff}] \\
\mathrm{RF}(\%)\end{array}$ & $\begin{array}{l}\Delta[\mathrm{HbT} \\
\mathrm{RF}(\% \\
\end{array}$ \\
\hline LWP475 & 0.21 & 0 & 122 & 111 & 106 \\
\hline LWP478 & 0.22 & 0 & 176 & 113 & 98 \\
\hline LWP479 & 1.03 & 1 & 69 & 113 & 3 \\
\hline LWP480 & 0.43 & 1 & 34 & 49 & 66 \\
\hline LWP481 & 0.26 & 0 & 86 & 103 & 104 \\
\hline LWP484 & 8.32 & 1 & 53 & 59 & 141 \\
\hline LWP485 & 0.18 & 0 & 111 & 100 & 96 \\
\hline LWP488 & 0.37 & 0 & 89 & 104 & 101 \\
\hline LWP489 & 22.64 & 1 & 34 & 75 & 102 \\
\hline LWP490 & 0.36 & 0 & 74 & 100 & 25 \\
\hline LWP491 & 0.14 & 0 & 117 & 123 & 92 \\
\hline LWP492 & 0.24 & 0 & 137 & 127 & 107 \\
\hline LWP493 & 0.22 & 0 & 113 & 95 & 50 \\
\hline LWP494 & 0.18 & 0 & 81 & 101 & 213 \\
\hline LWP495 & 0.43 & 1 & 74 & 95 & 117 \\
\hline LWP497 & 0.62 & 1 & 31 & 58 & 158 \\
\hline LWP498 & 0.31 & 0 & 88 & 104 & 113 \\
\hline LWP499 & 0.67 & 1 & 52 & 78 & 21 \\
\hline LWP500 & 0.48 & 1 & 74 & 100 & 100 \\
\hline LWP501 & 0.35 & 0 & 129 & 121 & 73 \\
\hline LWP502 & 0.90 & 1 & 79 & 107 & 194 \\
\hline LWP503 & 17.07 & 1 & 4 & 78 & 7 \\
\hline LWP504 & 112.07 & 1 & 42 & 82 & 52 \\
\hline LWP507 & 0.48 & 1 & 47 & 83 & 24 \\
\hline LWP509 & 1.00 & 1 & 57 & 58 & 109 \\
\hline LWP511 & 0.31 & 0 & 99 & 117 & 119 \\
\hline LWP512 & 0.29 & 0 & 87 & 115 & 136 \\
\hline
\end{tabular}

fraction of $\leq 79 \%$ for the $\Delta[$ oxCCO] signal within 30 min post $\mathrm{HI}$ is indicative of a severe brain injury at $24 \mathrm{~h}$ with $100 \%$ sensitivity and $93 \%$ specificity.

The cut-off recovery fractions of $\Delta[\mathrm{oxCCO}], \Delta[\mathrm{HbDiff}]$, and $\Delta[\mathrm{HbT}]$ with their corresponding sensitivity and specificity graphs are presented in Fig. 3.

Figure 4 illustrates the measurements from miniCYRIL, ${ }^{1} \mathrm{H}$, and ${ }^{31} \mathrm{P}-\mathrm{MRS}$, as well as the systemic data for all the piglets 

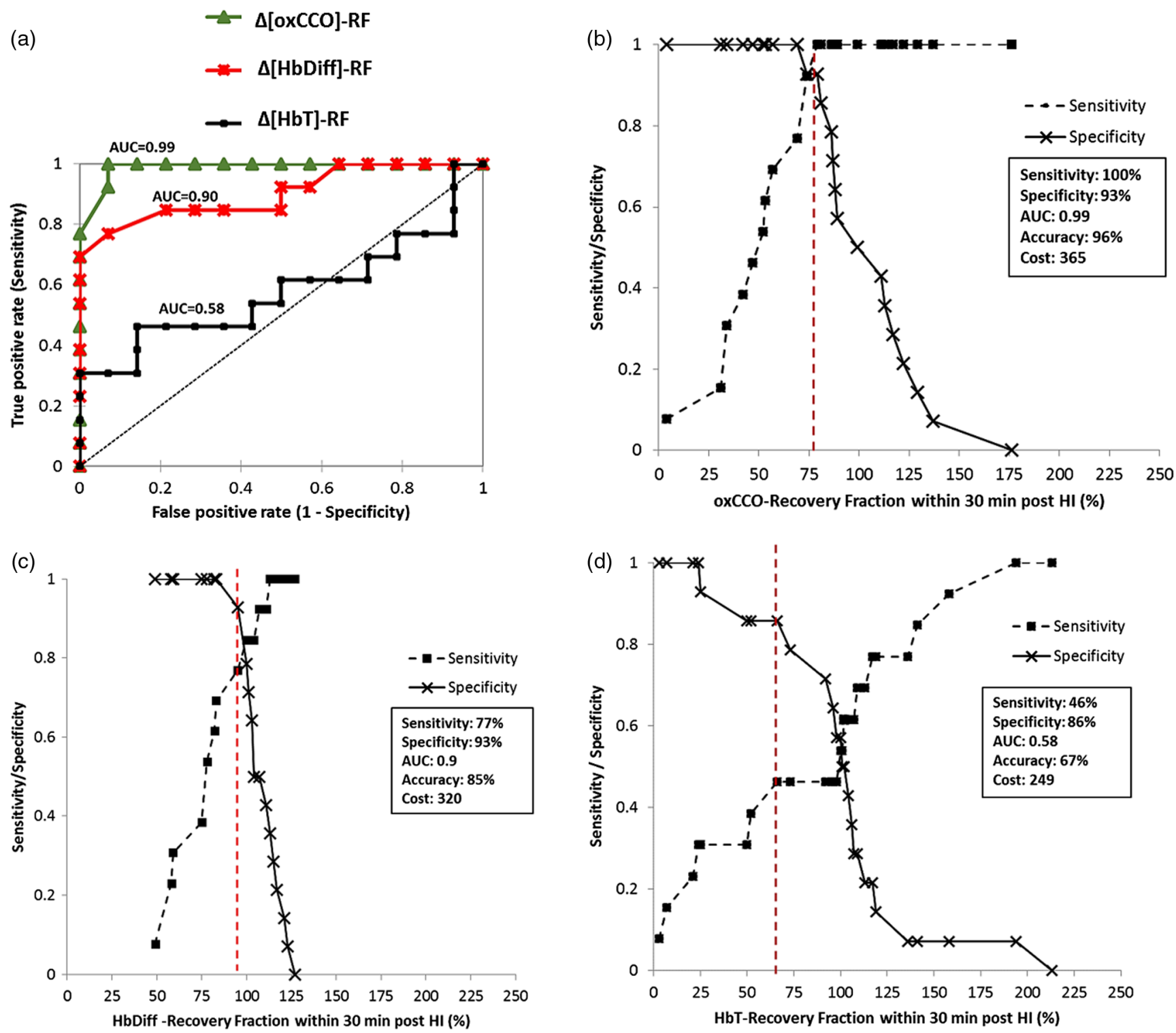

Fig. 3 (a) ROC curves for the recovery fractions of $(\Delta[\mathrm{HbT}]), \Delta[\mathrm{HbDiff}]$, and $\Delta[\mathrm{oxCCO}]$. (b) AUC of the $\Delta$ [oxCCO]-RF is 0.99 and the test is $96 \%$ accurate with $100 \%$ sensitivity and $93 \%$ specificity. (c) The AUC of the $\Delta[\mathrm{HbDiff}]-\mathrm{RF}$ is 0.9 and the test is $85 \%$ accurate for sensitivity and specificity of $77 \%$ and $93 \%$, respectively. (d) The test for $\Delta[\mathrm{HbT}]-\mathrm{RF}$ has the least accuracy $(67 \%)$ and AUC $(0.58)$ with poor sensitivity (46\%) and specificity (86\%). The threshold recovery fraction for each bNIRS signal is marked with a dotted line.

within mild and severe injury groups classified based on $\Delta[\mathrm{oxCCO}]-\mathrm{RF}$ (results are summarized in Table 4). In none of the piglets in severe injury group, the recovery fraction of $\Delta[\mathrm{oxCCO}]$ was restored to baseline $(100 \%)$ within $30 \mathrm{~min}$ post HI [Fig. 4(a)] and the recovery fraction in this group was on average $58 \%$ smaller than that of the mild injury group $(p<$ $0.0001)$. Even though the cerebral oxygenation $(\Delta[$ HbDiff $])$ in some piglets in the severe injury group recovered back to baseline [Fig. 4(b)], the average recovery fraction in this group was around $30 \%$ smaller than that of the mild injury group $(p=0.0001)$. There was no significant difference in the recovery of cerebral blood volume $\Delta[\mathrm{HbT}]$ in both groups [Fig. 4(c)]. However, piglets identified with severe injury at $24 \mathrm{~h} \mathrm{had} \mathrm{a} \mathrm{sig-}$ nificantly higher HR than the mild injury group within $30 \mathrm{~min}$ post $\mathrm{HI}(\Delta \mathrm{HR}=33 \mathrm{bpm}, p=0.05)$.
${ }^{1} \mathrm{H}$-MRS-measured metabolites were significantly different between groups at $24 \mathrm{~h}$. On average, piglets in the severe injury category had significantly higher thalamic and white matter Lac/NAA than the group with mild injury [ $\Delta \log$-thal (Lac/ $\mathrm{NAA})=0.5, p<0.0001$ and $\Delta \log -\mathrm{wm}(\mathrm{Lac} / \mathrm{NAA})=0.7$, $p=0.0001]$.

The average ${ }^{31} \mathrm{P}-\mathrm{MRS}$-measured $\mathrm{pH}$ in the severe injury group was also more acidic $(\Delta \mathrm{pH}=0.1, p=0.01)$, but no significant difference was observed in other metabolites measured this way between mild and severe injury groups as classified by $\Delta[\mathrm{oxCCO}]-\mathrm{RF}$ at $24 \mathrm{~h}$. However, at $48 \mathrm{~h}$, piglets with $\Delta[\mathrm{oxCCO}]-\mathrm{RF}$ of $\leq 79 \%$ (severe injury) had significantly lower PCr/epp (0.07, $p=0.003$ ) and higher Pi/epp (0.1, $p=0.01)$. Furthermore, at $48 \mathrm{~h}$, piglets in the severe injury group had further elevated Lac/NAA in both thalamus and white matter 
(a)

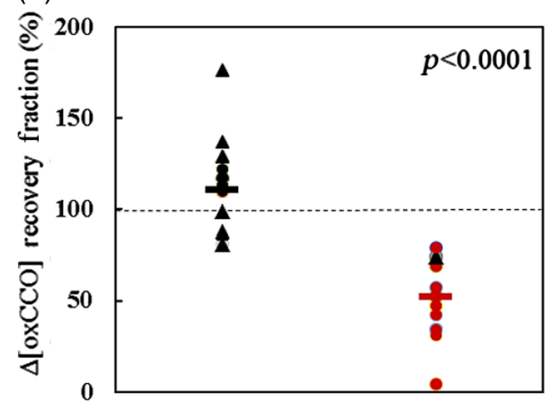

(d) Thalamus Lac/NAA
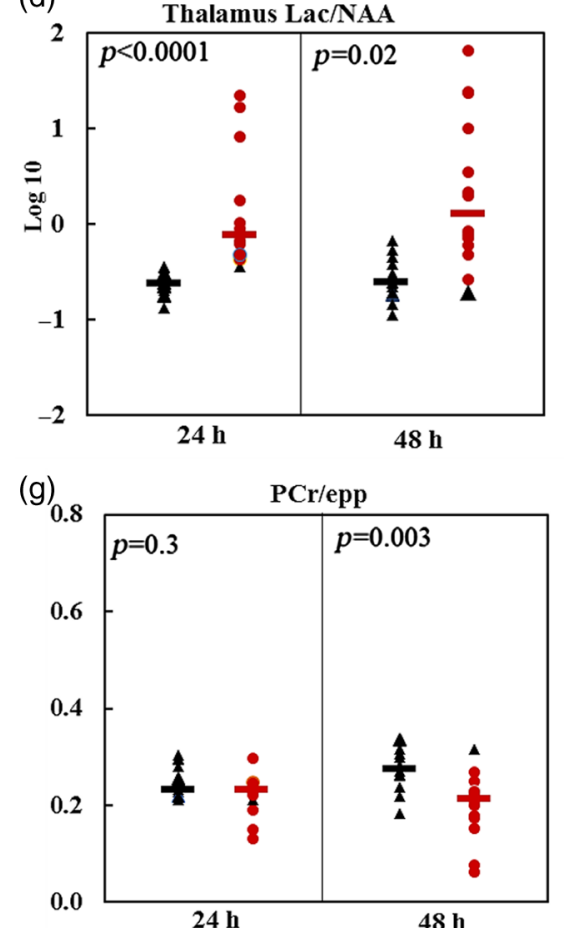

(j)

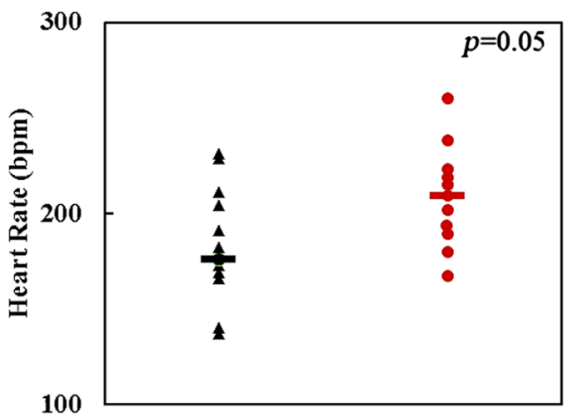

(b)

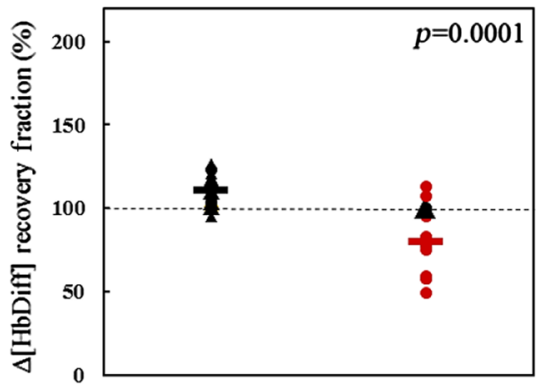

(e)

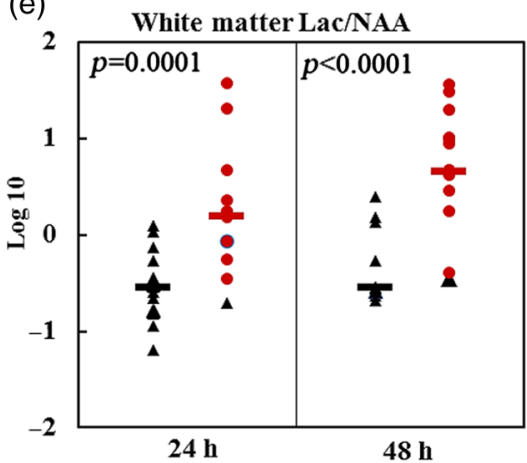

(h)

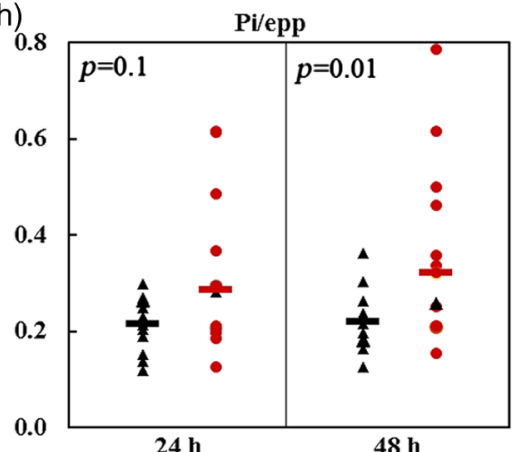

(k)

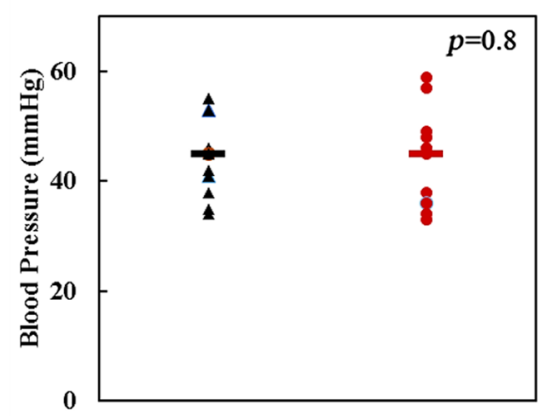

(c)

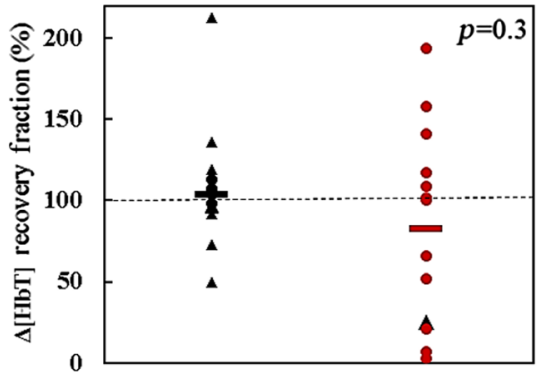

(f)

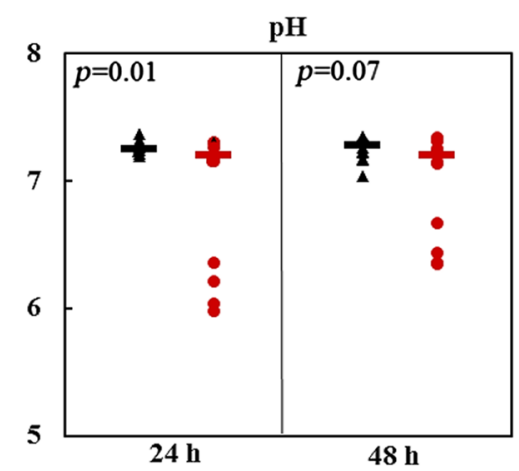

(i)

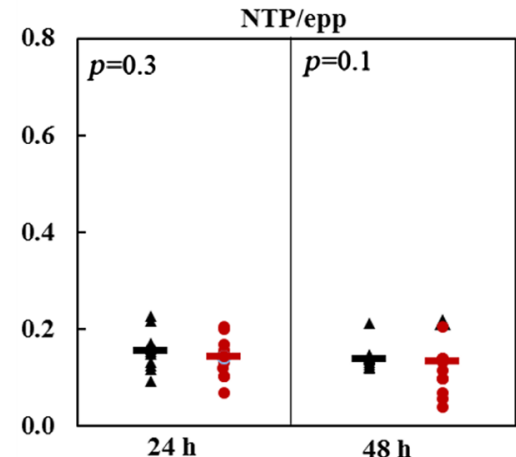

(I)

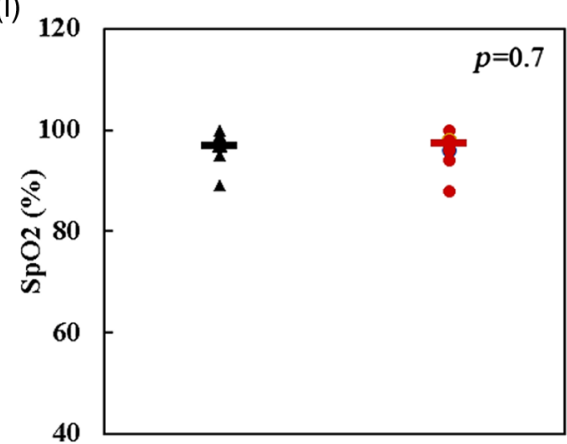

Fig. 4 Classification of piglets in two groups of mild ( $\Delta[0 \mathrm{oxCO}]-\mathrm{RF}>79 \%)$ and severe $(\Delta[\mathrm{oxCCO}]-\mathrm{RF}$ $\leq 79 \%$ ) injury as defined by $\Delta$ [oxCCO]-RF. The red dots and black triangle markers are the true positive (24-h thalamic Lac/NAA $\geq 0.39$ ) and true negatives (24-h thalamic Lac/NAA <0.39), respectively. (a)-(c) Recovery fraction of the bNIRS signals in piglets classified in the mild and the severe injury groups based on the threshold recovery fraction of $\Delta[$ oxCCO] $(79 \%)$. The dotted line marks a $100 \%$ recovery. (d) and (e) ${ }^{1} \mathrm{H}-\mathrm{MRS}$ measurements at 24 and $48 \mathrm{~h}$ post $\mathrm{HI}$ in piglets with mild and severe injuries. (f)-(i) ${ }^{31} \mathrm{P}$-MRS measurements at 24 and $48 \mathrm{~h}$ post $\mathrm{HI}$ in piglets with mild and severe injuries. (j)-(I) Systemic data are averaged over a 1-min window at recovery (when the recovery fraction of miniCYRIL signals were established). 
Table 4 Baseline and recovery values of bNIRS-RF, systemic data, as well as 24 and $48 \mathrm{~h}$ MRS measurements for piglets categorized based on $\Delta[$ oxCCO $]-R F$. Data are presented in median \pm IQR.

\begin{tabular}{|c|c|c|c|c|}
\hline bNIRS (recovery fraction) & \multicolumn{2}{|c|}{ Mild $($ oxCCO $>79 \%) n=14$} & \multicolumn{2}{|c|}{ Severe $(\mathrm{oxCCO} \leq 79 \%) n=13$} \\
\hline $\operatorname{oxCCO}(\%)$ & \multicolumn{2}{|c|}{$111 \pm 34$} & \multicolumn{2}{|c|}{$53 \pm 37^{\star *}$} \\
\hline HbDiff (\%) & \multicolumn{2}{|c|}{$111 \pm 14$} & \multicolumn{2}{|c|}{$80 \pm 36^{* *}$} \\
\hline $\mathrm{HbT}(\%)$ & \multicolumn{2}{|c|}{$104 \pm 17$} & \multicolumn{2}{|c|}{$83 \pm 91$} \\
\hline Systemic & Baseline & Recovery & Baseline & Recovery \\
\hline $\mathrm{SpO}_{2}(\%)$ & $96 \pm 2$ & $97 \pm 2$ & $95 \pm 4$ & $98 \pm 2$ \\
\hline HR (bpm) & $181 \pm 41$ & $176 \pm 35$ & $192 \pm 19$ & $209 \pm 30^{*}$ \\
\hline Blood pressure (mmHg) & $50 \pm 6$ & $45 \pm 12$ & $53 \pm 8$ & $45 \pm 13$ \\
\hline${ }^{1} \mathrm{H}-\mathrm{MRS}$ & $24 \mathrm{~h}$ & $48 \mathrm{~h}$ & $24 \mathrm{~h}$ & $48 \mathrm{~h}$ \\
\hline Thalamus Lac/NAA (log 10) & $-0.62 \pm 0.2$ & $-0.61 \pm 0.3$ & $-0.11 \pm 1.1^{\star \star}$ & $0.12 \pm 1.1^{*}$ \\
\hline White matter Lac/NAA $(\log 10)$ & $-0.55 \pm 0.5$ & $0.3 \pm 0.7$ & $0.19 \pm 0.6^{\star *}$ & $0.66 \pm 0.5^{\star \star}$ \\
\hline $\mathrm{pH}$ & $7.3 \pm 0.1$ & $7.3 \pm 0.1$ & $7.2 \pm 0.7^{\star \star}$ & $7.2 \pm 0.5$ \\
\hline${ }^{31}$ P-MRS & $24 \mathrm{~h}$ & $48 \mathrm{~h}$ & $24 \mathrm{~h}$ & $48 \mathrm{~h}$ \\
\hline PCr/epp & $0.23 \pm 0.1$ & $0.28 \pm 0.1$ & $0.23 \pm 0.03$ & $0.21 \pm 0.1^{\star \star}$ \\
\hline Pi/epp & $0.21 \pm 0.1$ & $0.22 \pm 0.1$ & $0.29 \pm 0.1$ & $0.32 \pm 0.2^{\star \star}$ \\
\hline NTP/epp & $0.16 \pm 0.03$ & $0.14 \pm 0.01$ & $0.14 \pm 0.04$ & $0.13 \pm 0.04$ \\
\hline
\end{tabular}

$[\Delta$ log-thal $(\mathrm{Lac} / \mathrm{NAA})=0.7, p=0.02$ and $\Delta \log -\mathrm{wm}(\mathrm{Lac} /$ $\mathrm{NAA})=0.4, p<0.0001]$.

\section{Discussion}

We have developed a cost-effective miniature bNIRS system, called miniCYRIL, based on a miniature white-light source and a customized solution of a miniature spectrometer by Ocean Optics and Wasatch Photonics. The miniCYRIL system can make transmission-mode brain measurements in the piglet from 600 to $1000 \mathrm{~nm}$ with SDS of up to $45 \mathrm{~mm}$ (depending on the head diameter) and spectral resolution of $4 \mathrm{~nm}$. The miniCYRIL acquired full spectral data in transmission mode through the piglets' heads with a sampling rate of $0.1 \mathrm{~Hz}$ and using the spectral data estimated brain tissue concentration changes of $\mathrm{HbO}_{2}, \mathrm{HHb}$, and oxCCO via a measured optical pathlength in real time.

In this study, we did not detect any light-induced thermal effects or damages to the piglets since the light source emits low-power noncoherent light with very small irradiances at tissue interface $\left(1.92 \mathrm{~mW} / \mathrm{cm}^{2}\right.$ across all wavelengths, which becomes much smaller as light travels through the tissue due to the use of highly diverging large NA optical fibers). This is far less than the average irradiance delivered by the entire NIR part of sunlight on a sunny day that is more than $10 \mathrm{~mW} /$ $\mathrm{cm}^{2} .{ }^{60,61}$ In addition, the possibility of stimulating photobiological effects in piglets' brains was also highly unlikely in these experiments, because we observed a stable baseline in the chromophore concentration signals in all piglets. Furthermore, in photobiomodulation, the effective irradiance is typically around tens to a few hundred $\mathrm{mW} / \mathrm{cm}^{2}$, which is produced by high-fluence monochromatic/quasimonochromatic light from lasers or LEDs in the red/NIR region, ${ }^{62-64}$ in contrast with the broad spectrum multidirectional light produced by the lowpower halogen lamp that was used in this study.

The retrofit cooling upgrade in the Ventana spectrometer (keeping the $\mathrm{CCD}$ temperature at $15^{\circ} \mathrm{C}$ ) reduced the dark count by $75 \%$ and improved the stability of the spectrometer during the measurement. This is specifically beneficial during long measurements (few hours) since in differential spectroscopy high unstable dark noise on the spectral data leads to inaccurate attenuation change measurements and ultimately erroneous concentration data.

We performed a feasibility study to assess the physiological significance of the measurements produced by miniCYRIL in determining an early marker for classifying the outcome (prediction of brain injury severity) in the neonatal piglet following HI. Previously, HI was induced inside an MR scanner and ${ }^{31} \mathrm{P}-$ MRS-measured $\beta$-NTP peak height was used to control the HI and determine the extent of the recovery in piglets. ${ }^{10}$ Despite its useful measurements, using MRS to induce HI and examine the severity of the primary injury has some limitations including the cost and the need for a trained MR physicist, as well as the fact 
that clinicians do not have access to the piglet during the procedure in case of emergencies. Located at the piglet's cotside, miniCYRIL can be easily operated by clinicians to monitor brain hemodynamics and oxygenation changes, as well as metabolic status during $\mathrm{HI}$ and recovery at up to $48 \mathrm{~h}$.

During the HI insult, mitochondrial respiration is inhibited due to significant reduction in blood supply (occlusion of both common carotid arteries) and the unavailability of oxygen as the final electron acceptor in the electron transport chain (ETC) $\left(\mathrm{FiO}_{2}=6 \%\right)$, which consequently leads to a reduction in the $\Delta[$ oxCCO] signal (CCO being the final protein complex in the ETC). After the resuscitation, oxygen and brain blood flow are recovered back to baseline. Hüttemann et al. ${ }^{65}$ highlighted that there are three distinct responses/phases for CCO and cytochrome-c in ischemia/reperfusion injury: (1) the ischemic starvation phase, (2) the reperfusion-induced hyperactivation phase, and (3) the mitochondrial dysfunction phase. We observed phase 1 in all our animals demonstrated by the significant reduction in [oxCCO]. In many of our animals (see Figs. 4 and 8, and Sec. 8), we observed a hyperemia effect and hyperactivation phase following reperfusion as quantified by the recovery fraction of $\Delta[\mathrm{HbDiff}]$ and $\Delta[\mathrm{oxCCO}]$ above $100 \%$ (above baseline). In a significant number of the piglets, we noted the absence of phase 2 and instead we detected an early and prolonged mitochondrial dysfunction phase following the HI starvation phase [see an example in Figs. 1(d) and 1(e)]. This observation is independent of the initial $\mathrm{HI}$ injury as all animals received the same insult. We have recently investigated this observation using our system's biology computational model ${ }^{66}$ and concluded that this early and prolonged nonrecovery of oxCCO and tissue energetics could be due to multiple effects that involve mitochondrial uncoupling, disrupted brain blood flow, and cell death. Therefore, our current results are consistent with the indication of oxCCO inhibition playing a major role in brain cell death during neonatal HI, as discussed previously by Cooper and Springett. ${ }^{32}$

Our results demonstrate that piglets with oxCCO signal recovery of $\leq 79 \%$ within $30 \mathrm{~min}$ after $\mathrm{HI}$ had a more severe brain injury as shown by $24-\mathrm{h}{ }^{1} \mathrm{H}$-MRS-measured thalamic Lac/NAA, a robust marker of neurodevelopmental outcome. ${ }^{15}$ Piglets with $\Delta[$ oxCCO $]-R F$ of $\leq 79 \%$ also had significantly lower $\mathrm{PCr}$ /epp and higher Pi/epp at $48 \mathrm{~h}$ post $\mathrm{HI}$, as measured by ${ }^{31} \mathrm{P}-\mathrm{MRS}(p<0.01)$. As reported previously by Bainbridge et al., ${ }^{10}$ an elevated Pi level is a characteristic of HIE (Pi being a product of ATP hydrolysis) and a decreased $\mathrm{PCr}$ (as a reservoir for high-energy phosphate) is a marker of the anaerobic condition. These changes are more prominent after $48 \mathrm{~h}$ due to secondary energy failure. ${ }^{54}$

It was demonstrated previously in our preclinical model, using a former in-house developed bNIRS instrument, that there is a strong relationship between the oxCCO and ${ }^{31} \mathrm{P}-\mathrm{MRS}$ ratios during $\mathrm{HI}$ and recovery, and piglets that survived to $48 \mathrm{~h}$ of the experiment had greater recovery of their ${ }^{31} \mathrm{P}-\mathrm{MRS}$ ratios and $\Delta$ [oxCCO $]$ at $1 \mathrm{~h}$ post $\mathrm{HI} .{ }^{10}$ In this study, piglets were categorized into two groups of mild and severe injuries based on the recovery fraction of $\Delta$ [oxCCO] within $30 \mathrm{~min}$ post $\mathrm{HI}$ (threshold $79 \%$, sensitivity $100 \%$, specificity $93 \%$ ). Piglets in the severe injury group had significantly higher Lac/NAA (measured in thalamus and white matter) at $24 \mathrm{~h}$ post $\mathrm{HI}(p<0.01)$. The Lac/NAA measurement was still significantly greater in the severe injury group (as defined by RF- $\Delta[$ oxCCO $]$ ) at $48 \mathrm{~h}$ post $\mathrm{HI}$ (thalamus $p<0.05$, white matter $p<0.01$ ). The
MRS-derived $\mathrm{pH}$ was also significantly lower in piglets with $\Delta$ oxCCO]-RF of $\leq 79 \%$ (severe injury group) at $24 \mathrm{~h}$.

The recovery fraction of $\Delta[\mathrm{oxCCO}]$ in piglets with severe injury was on average around $60 \%$ smaller than that of the mild injury group, and this was not associated with any interventions or treatment, as the therapeutic hypothermia and randomized treatments were administered $1 \mathrm{~h}$ post HI. Piglets with recovery fraction of $\Delta[\mathrm{oxCCO}] \leq 79 \%$ had also significantly lower recovery fraction of blood oxygenation ([ $\Delta$ HbDiff $]-R F \sim 33 \%$, $p<0.01$ ) even though there was no difference in systemic oxygenation $\left(\mathrm{SpO}_{2}\right)$ between groups. The severe injury group had on average $30 \%$ smaller recovery fraction of total blood volume $\Delta[\mathrm{HbT}]-\mathrm{RF}$, which was not statistically significant as both groups had a wide range of recovery in their $\Delta[\mathrm{HbT}]$. While blood pressure recovered similarly in piglets, regardless of their category $(\sim 45 \pm 12 \mathrm{mmHg}), \mathrm{HR}$ in piglets with severe injury was on average $\sim 30 \mathrm{bpm}$ higher than in piglets with mild brain injury $(p<0.05)$.

All the piglets in the study received hypothermia treatment at $1 \mathrm{~h}$ following $\mathrm{HI}$ and some piglets also received adjacent therapies, including magnesium, melatonin, and ethanol. Our analysis of the bNIRS measurements was done before the administration of any therapies and, as such, we did not expect any treatment effects. In addition, we have not observed any association between our bNIRS recovery fraction analysis and treatment procedure. We did not expect to see such associations as the original piglet study was not powered for that purpose. To investigate this further, we are planning a future study that uses the bNIRS recovery fraction of [oxCCO] within 30 min following HI to guide treatment strategies at $1 \mathrm{~h}$ following $\mathrm{HI}$.

The recovery fraction analysis provides a potential real-time marker (being the recovery fraction of the oxCCO signal) to predict the severity of the injury as early as $30 \mathrm{~min}$ post HI. Therefore, miniCYRIL can provide valuable information in preclinical studies to assess the severity of HI, predict the outcome, and investigate possible treatment strategies. The limitation of this analysis is that it is performed under controlled experimental condition and hence cannot be applied in the same way in hospital to assess the severity of the primary injury in babies affected with HI, as the time of the HI is unknown in the neonates and hence we do not have access to a baseline value. In view of this issue, our group is currently working toward developing methodologies in order to implement absolute oxCCO measurement in the clinical setting as it gives a clearer understanding of tissue metabolism and allows easy comparison between patients without the need for complicated data analyses.

The miniCYRIL based on a customized Ventana spectrometer might have some advantages over previously described miniature systems based on the QE65000 or more recently the QE65 Pro, which have been used for reflection mode brain measurements in piglets, adults, and rats. ${ }^{40-42,44-47}$ The combination of larger throughput spectrograph $(F / 2)$ with larger NA optical fibers $(0.57)$ in miniCYRIL enhances light collection capability of the detection unit and allows performing measurement at large SDS while maintaining the measurement accuracy. This is crucial for assessing brain metabolism since computational and functional activation studies have shown that $\mathrm{CCO}$ is a depth-dependent signal (due to mitochondria being more abundant in deeper tissue layers) and hence its changes can be more accurately measured at larger SDS due to the allowance 
of light to traverse the brain with greater penetration depth before reaching the detector. ${ }^{35,39,67,68}$ In this study, optodes were positioned on the left and right sides of the head at approximately the middle part of the brain enabling transmission mode (whole brain) measurement, which is more sensitive to deeper structures compared to reflection mode. ${ }^{69}$

Other studies that have used QE-based spectrometers $(F / 4)$ are limited to smaller throughput, despite the improvements that were made in light collection capability of the system as a result of modifying detector fiber's geometry ${ }^{41}$ or using a high NA fiber (0.5) instead of the standard NA $=0.22$, which is adapted for the QE65 Pro. ${ }^{47}$ We have found that utilizing a large NA detector fiber with QE65 Pro in piglets leads to a significant amount of photon loss and noise in forms of stray light inside the spectrometer, which is due to the large mismatch between the NA of the fiber and the $f$-number of the spectrometer (the large NA of the detector fiber drastically overfills the small acceptance cone of the miniature spectrometer). ${ }^{43}$ In this study, we used an optimized solution where a large NA fiber is used with a low $f$-number miniature spectrometer (Ventana, $F / 2$ ) which maximizes efficient light collection in transmission mode despite having a less sensitive $\mathrm{CCD}^{43}$ (Table 1).

Another important characteristic of miniCYRIL is the onboard data processing and real-time display of the concentration data, which is a requirement for use in the clinical environment. Furthermore, this system enables real-time measurement of optical pathlength through the brain. This feature improves the estimation of chromophore concentration, which is especially crucial when there are changes in the nature and anatomy of the brain tissue (e.g., through swelling or edema) during the measurement period. ${ }^{70}$

\section{Conclusion}

We have developed and described a miniature bNIRS system (miniCYRIL) based on a miniature white-light source and a customized miniature spectrometer. The system was utilized in a preclinical study of neonatal encephalopathy to measure changes in cerebral oxygenation, hemodynamics, and metabolism in real time in 27 piglets induced with $\mathrm{HI}$.

We have developed an algorithm to use miniCYRIL for quantification of HI brain tissue injury severity (mild and severe injury). Recovery fraction of the oxCCO signal within $30 \mathrm{~min}$ post $\mathrm{HI}$ demonstrated to be a good predictive marker for the severity of brain injury in piglets with $100 \%$ sensitivity and 93\% specificity $(\mathrm{AUC}=0.99)$. Piglets with $\Delta[\mathrm{oxCCO}]-\mathrm{RF}$ of $\leq 79 \%$ had significantly smaller cerebral oxygenation (HbDiff) recovery at $30 \mathrm{~min}$ post $\mathrm{HI}$ and higher thalamic and white matter Lac/NAA after 24 and $48 \mathrm{~h}$. The MRS-measured $\mathrm{pH}$ in this group was more acidic at $24 \mathrm{~h}$ and they had a significantly higher HR within 30-min recovery period. At 48 h, when secondary energy failure is expected to be more prominent, piglets in the severe injury group had significantly lower ${ }^{31} \mathrm{P}-\mathrm{MRS}$-measured $\mathrm{PCr} / \mathrm{epp}$ and higher Pi/epp, which are characteristics of an HI brain.

The miniCYRIL system can be a cost-effective solution in preclinical studies for monitoring $\mathrm{HI}$ and assessing treatment strategies, as it can offer a brain injury prediction within $30 \mathrm{~min}$ after HI. The proposed instrument can be operated by experimentalists and clinical staff without the need for a dedicated technical team. Currently (in 2019), the cost of miniCYRIL components is about $£ 20,000$ to $£ 25,000$, which is marginal compared to the components cost for an MR machine that is approximately $£ 1$ million or more.

Finally, with a much smaller footprint than the custom-made bNIRS systems, miniCYRIL can be utilized more effectively in the hospital, both in the intensive care unit and/or in the operation theater.

\section{Appendix A}

\subsection{Dark Measurement}

Dark intensity measurements across 780 to $900 \mathrm{~nm}$ at time 0 and after $10 \mathrm{~h}$ are presented in Fig. 5, before and after the customization (retrofit cooling) of Ventana VIS-NIR. The distribution of intensity across all the wavelengths in Ventana has a flat shape with no prominent features. However, the mean dark intensity across all the wavelengths is significantly large in both cases when there is no light input into the spectrograph, being $8850 \pm 725$ counts at time 0 with $32 \%$ increase in the dark count $(11672 \pm 897)$ across all wavelengths after $10 \mathrm{~h}$ [Fig. 5(a)].

Furthermore, the intensity changes at one wavelength $(\lambda=780 \mathrm{~nm})$ over time is significantly high and has a mean value of $11297 \pm 1020$ counts. The considerably large standard deviation from the mean dark intensity at $780 \mathrm{~nm}$ shows large variability in the measurement, which is a limiting factor, and leads to erroneous concentration change measurements.

Figure 5(c) shows dark noise across all wavelengths (780 to $900 \mathrm{~nm}$ ) after the retrofit cooling customization in Ventana VIS-NIR spectrometer at time 0 and after $10 \mathrm{~h}$. The mean intensity at time 0 is $2440 \pm 218$ when there is no light input into the spectrograph, which on average is $75 \%$ less than that produced by the original spectrometer. After $10 \mathrm{~h}$, the average increase in dark count across all wavelengths is almost negligible $(<1 \%)$, being $2472 \pm 223$ counts. Figure $5(d)$ shows the intensity change at one wavelength over time $(\lambda=780 \mathrm{~nm})$. The variation in the dark intensity has a mean value of $2625 \pm$ 25 counts over $10 \mathrm{~h}$, which is significantly smaller (77\%) than that in the absence of cooling. The considerably small standard deviation from the mean intensity at $780 \mathrm{~nm}$ over $10 \mathrm{~h}$ is highly significant and improves the accuracy of concentration measurement.

\subsection{System Stability}

Figure 6 shows the changes in the spectral data and chromophore concentrations resolved on a tissue-like phantom during dark (no light through the phantom) and when light was incident on the phantom at an SDS of $4 \mathrm{~cm}$.

There is a negligible change in the average dark intensity through the phantom across 780 to $900 \mathrm{~nm}$ and there are minor changes in the concentration data, which are stable with no drift for $2 \mathrm{~h}\left[\Delta[\mathrm{HHb}]=-0.15 \pm 0.16(\mu \mathrm{M} \times \mathrm{cm}), \Delta\left[\mathrm{HbO}_{2}\right]=\right.$ $-0.19 \pm 0.07(\mu \mathrm{M} \times \mathrm{cm}), \Delta[\mathrm{oxCCO}]=-0.14 \pm 0.07(\mu \mathrm{M} \times$ $\mathrm{cm})$ ]. Figures $6(\mathrm{c})$ and 6(d) show the spectral data and concentration changes on the phantom when the light was on continuously for $14 \mathrm{~h}$. The average change in the intensity of light traversing the phantom is around $8 \%$ and there is no drift in the concentration data showing the high stability of the miniCYRIL system over $14 \mathrm{~h}[\Delta[\mathrm{HHb}]=0.59 \pm 0.19(\mu \mathrm{M} \times \mathrm{cm})$, $\Delta\left[\mathrm{HbO}_{2}\right]=0.02 \pm 0.08 \quad(\mu \mathrm{M} \times \mathrm{cm}), \quad \Delta[\mathrm{oxCCO}]=-0.30 \pm$ $0.09(\mu \mathrm{M} \times \mathrm{cm})]$. 
Kaynezhad et al.: Quantification of the severity of hypoxic-ischemic brain injury in a neonatal preclinical model...

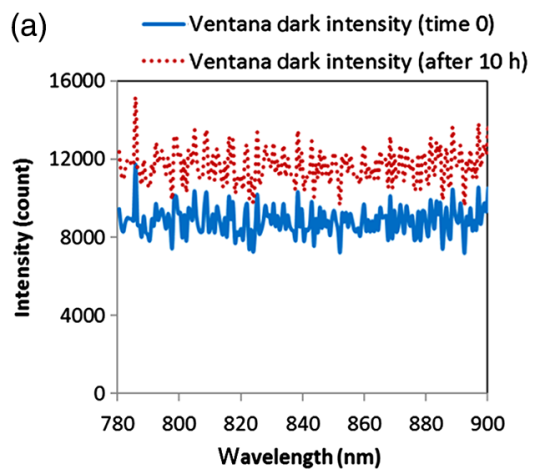

(b)

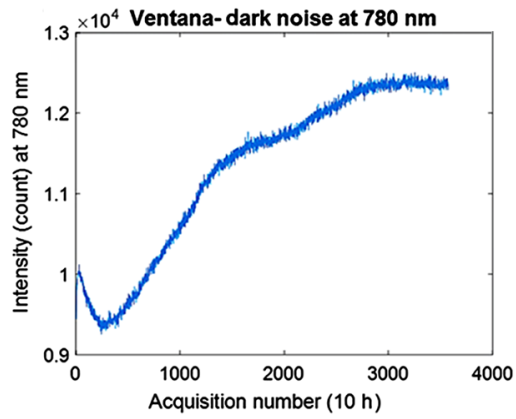

(c) - Ventana dark intensity with cooling (time 0 )

....... Ventana dark intensity with cooling (after $10 \mathrm{~h}$ )

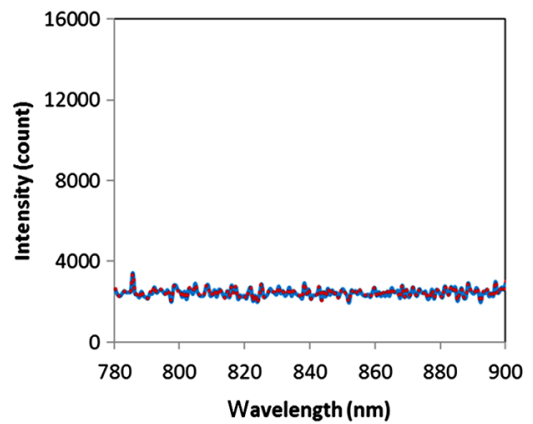

(d)

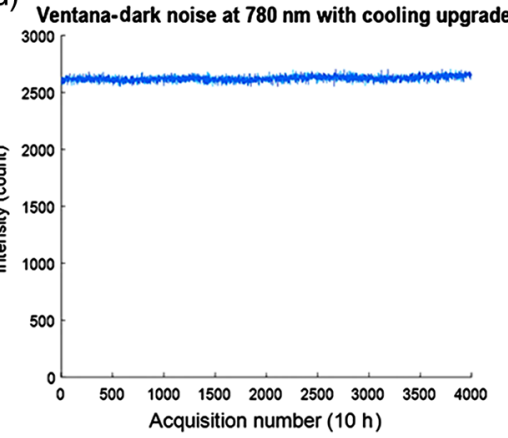

Fig. 5 (a) Dark intensity spectrum measured across 780 to $900 \mathrm{~nm}$ every $10 \mathrm{~s}$ when the slit of the spectrometer is closed at time 0 and after $10 \mathrm{~h}$. (b) The dark intensity changes at wavelength $780 \mathrm{~nm}$ over $10 \mathrm{~h}$. (c) Dark intensity spectrum measured across 780 to $900 \mathrm{~nm}$ every $10 \mathrm{~s}$ with the upgraded unit, the slit is closed at time 0 and after $10 \mathrm{~h}$. (d) The dark intensity changes at wavelength $780 \mathrm{~nm}$ over $10 \mathrm{~h}$.

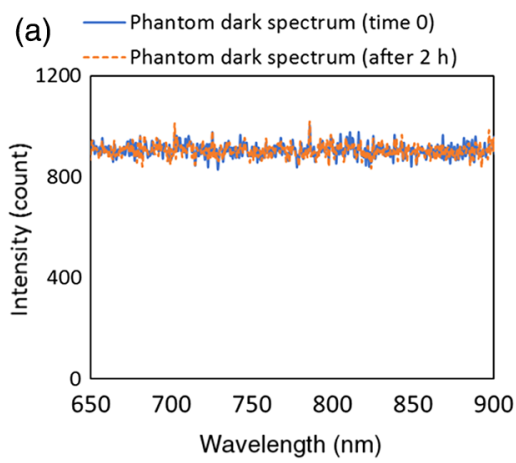

(b)
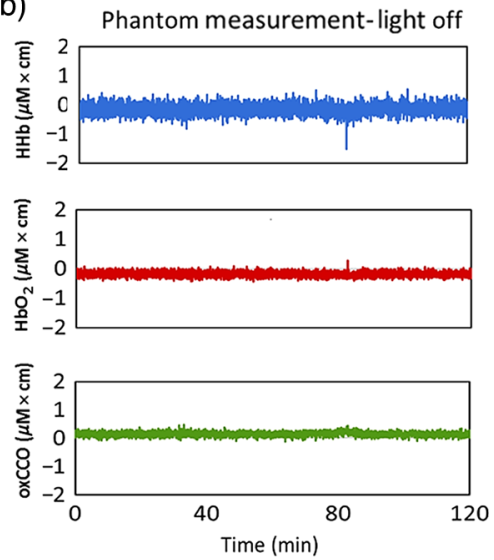

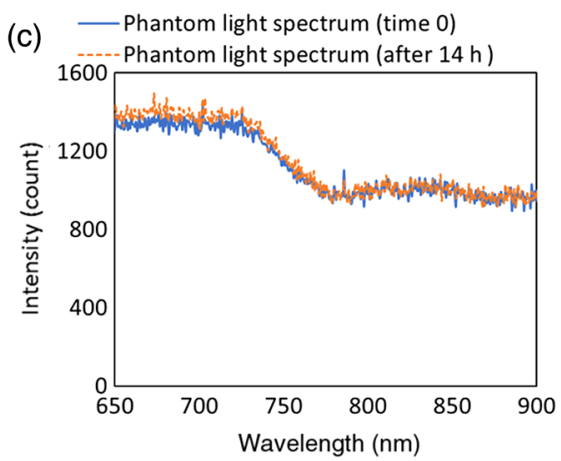

(d)
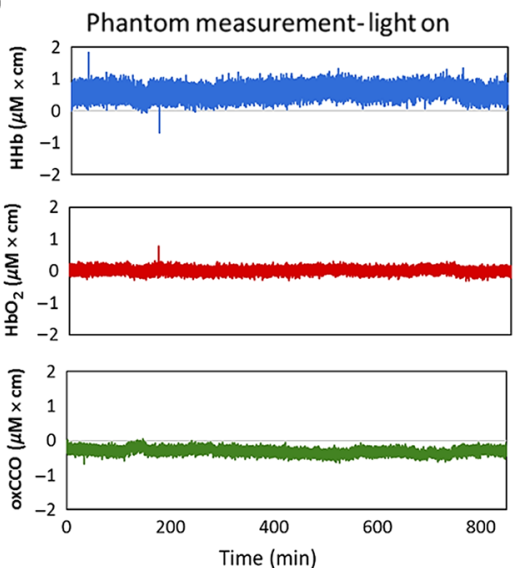

Fig. 6 Phantom measurements in dark and light showing the stability of the miniCYRIL system. (a) and (b) The spectral data and concentration changes in a tissue-like phantom ( $\mu a=0.02$ and $\mu^{\prime} s=0.9$, $\left[\mathrm{mm}^{-1} \mathrm{~mol}^{-1}\right]$ ) for $2 \mathrm{~h}$ when the shutter of the light source is closed. (c) and (d) The spectral data and concentration measurements for $14 \mathrm{~h}$, when the same phantom is illuminated (shutter open). Integration time for both measurements is $1 \mathrm{~s}$ and SDS is $4 \mathrm{~cm}$. 


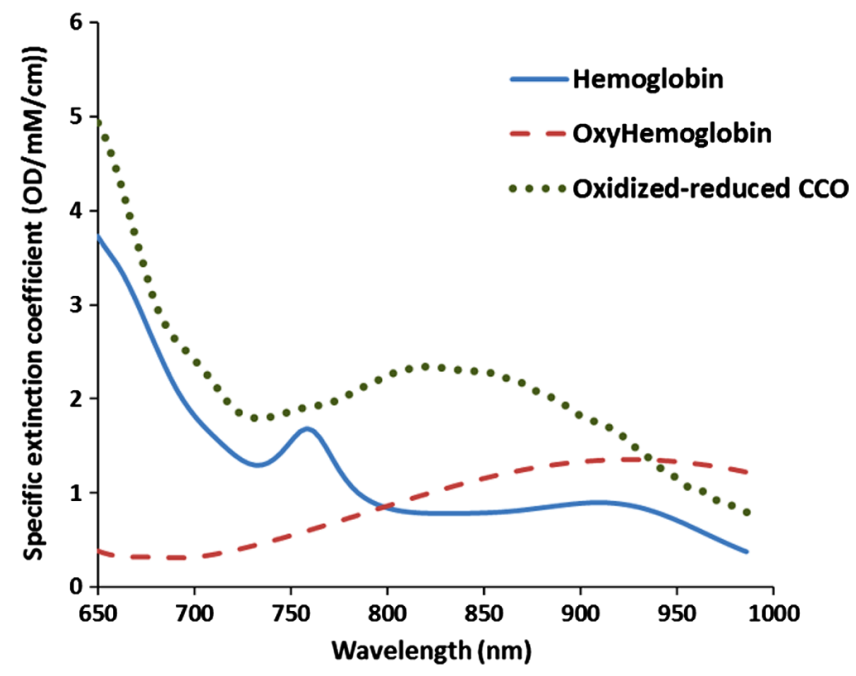

Fig. 7 The specific extinction coefficient of oxygenated and deoxygenated hemoglobin as well as the difference absorption spectrum of oxidized and reduced CCO. The data are taken from Ref. 71.

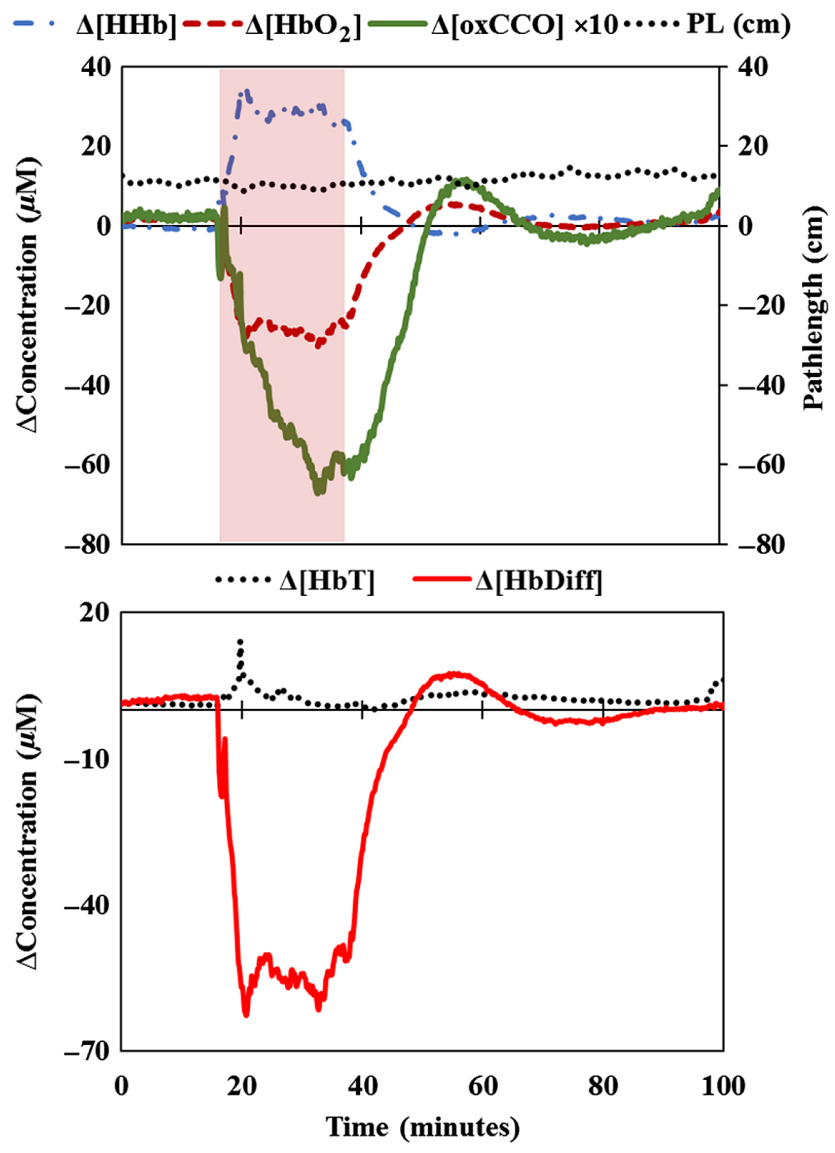

Fig. 8 An example of changes in the bNIRS measurements during baseline, HI, and recovery in a piglet (LWP498) as measured by miniCYRIL based on the second derivative spectroscopy technique for pathlength change measurement. The shaded area indicates the $\mathrm{HI}$ period. There is no change in the optical pathlength during $\mathrm{HI}$ and recovery. In this piglet, the HbDiff and oxCCO signals demonstrate a hyperemia and hyperactivation phase after $\mathrm{HI}$ (above baseline).

\section{Appendix B}

\subsection{Absorption Spectra of Chromophores}

Figure 7 shows the absorption spectra of oxygenated and deoxygenated hemoglobin as well as the difference absorption spectrum of oxidized and reduced CCO measured by Cope,${ }^{71}$ which was implemented in miniCYRIL's software [Eq. (1)] to calculate changes in the concertation of chromophores.

\section{Appendix C}

\subsection{Example of a Typical Response in Piglets with oxCCO-RF of $>79 \%$}

Figure 8 represents a typical response in piglets with $\Delta$ [oxCCO]-RF of $>79 \%$ following $\mathrm{HI}$ and being diagnosed with mild injury at $24 \mathrm{~h}$ post $\mathrm{HI}$ based on the thalamic ${ }^{1} \mathrm{H}-\mathrm{MRS}$ Lac/ NAA measurement. Following the initial ischemic starvation phase, we observe a reperfusion-induced hyperactivation phase.

\section{Disclosures}

The authors have nothing to disclose.

\section{Acknowledgments}

Funding support for this study was received from the UK Department of Health's NIHR BRC funding scheme and the Wellcome Trust (104580/Z/14/Z).

\section{References}

1. N. J. Robertson et al., "Melatonin as an adjunct to therapeutic hypothermia in a piglet model of neonatal encephalopathy: a translational study," Neurobiol. Dis. 121, 240-251 (2019).

2. L. Liu et al., "Global, regional, and national causes of under-5 mortality in 2000-15: an updated systematic analysis with implications for the sustainable development goals," Lancet 388(10063), 3027-3035 (2016).

3. S. A. Zanelli, D. A. Kaufman, and D. P. Stanley, "Hypoxic-ischemic encephalopathy: practice essentials, background, pathophysiology," Medscape, 2015, http://emedicine.medscape.com/article/973501overview (accessed 14 June 2015).

4. N. Nagdyman et al., "Early biochemical indicators of hypoxic-ischemic encephalopathy after birth asphyxia," Pediatr. Res. 49(4), 502-506 (2001).

5. E. C. Radlowski et al., "A neonatal piglet model for investigating brain and cognitive development in small for gestational age human infants," PLoS One 9(3), e91951 (2014).

6. E. B. Cady et al., "Phosphorus magnetic resonance spectroscopy $2 \mathrm{~h}$ after perinatal cerebral hypoxia-ischemia prognosticates outcome in the newborn piglet," J. Neurochem. 107(4), 1027-1035 (2008).

7. C. E. Cooper et al., "Use of mitochondrial inhibitors to demonstrate that cytochrome oxidase near-infrared spectroscopy can measure mitochondrial dysfunction noninvasively in the brain," J. Cereb. Blood Flow Metab. 19(1), 27-38 (1999).

8. M. Ezzati et al., "Dexmedetomidine combined with therapeutic hypothermia is associated with cardiovascular instability and neurotoxicity in a piglet model of perinatal asphyxia," Dev. Neurosci. 39(1-4), 156-170 (2017).

9. K. M. Tichauer et al., "Cerebral metabolic rate of oxygen and amplitude-integrated electroencephalography during early reperfusion after hypoxia-ischemia in piglets," J. Appl. Physiol. 106(5), 1506-1512 (2009).

10. A. Bainbridge et al., "Brain mitochondrial oxidative metabolism during and after cerebral hypoxia-ischemia studied by simultaneous phosphorus magnetic-resonance and broadband near-infrared spectroscopy," Neuroimage 102(P1), 173-183 (2014). 
11. P. L. Hope et al., "Cerebral energy metabolism studied with phosphorus NMR spectroscopy in normal and birth-asphyxiated infants," Lancet 2(8399), 366-370 (1984).

12. D. Azzopardi et al., "Prognosis of newborn infants with hypoxic-ischemic brain injury assessed by phosphorous magnetic resonance spectroscopy," Pediatr. Res. 25(5), 445-451 (1989).

13. F. Groenendaal et al., "Cerebral lactate and N-acetyl-aspartate/choline ratios in asphyxiated full-term neonates demonstrated in vivo using proton magnetic resonance spectroscopy," Pediatr. Res. 35(2), 148-151 (1994).

14. L. S. De Vries and F. Groenendaal, "Patterns of neonatal hypoxicischaemic brain injury," Neuroradiology 52(6), 555-566 (2010).

15. S. Mitra et al., "Proton magnetic resonance spectroscopy lactate/Nacetylaspartate within 2 weeks of birth accurately predicts 2 -year motor, cognitive and language outcomes in neonatal encephalopathy after therapeutic hypothermia," Arch. Dis. Child. - Fetal Neonatal Ed. 104, F424-F432 (2019).

16. S. J. Matcher et al., "Performance comparison of several published tissue near-infrared spectroscopy algorithms," Anal. Biochem. 227(1), 54-68 (1995).

17. G. Bale, C. E. Elwell, and I. Tachtsidis, "From Jöbsis to the present day: a review of clinical near-infrared spectroscopy measurements of cerebral cytochrome-c-oxidase," J. Biomed. Opt. 21(9), 091307 (2016).

18. I. Tachtsidis et al., "In-vivo measurements of brain haemodynamics and energetics using multimodal spectroscopy in perinatal hypoxia-ischaemia," in Biomed. Opt. and 3-D Imaging, p. JM3A.27 (2012).

19. R. Springett et al., "Oxygen dependency of cerebral oxidative phosphorylation in newborn piglets," J. Cereb. Blood Flow Metab. 20(2), 280-289 (2000).

20. M. M. Tisdall et al., "Near-infrared spectroscopic quantification of changes in the concentration of oxidized cytochrome c oxidase in the healthy human brain during hypoxemia," J. Biomed. Opt. 12(2), 024002 (2007).

21. M. M. Tisdall et al., "Increase in cerebral aerobic metabolism by normobaric hyperoxia after traumatic brain injury," J. Neurosurg. 109(3), 424-432 (2008).

22. I. Tachtsidis et al., "A hybrid multi-distance phase and broadband spatially resolved spectrometer and algorithm for resolving absolute concentrations of chromophores in the near-infrared light spectrum," Adv. Exp. Med. Biol. 662, 169-175 (2010).

23. A. Ghosh et al., "Use of a hybrid optical spectrometer for the measurement of changes in oxidized cytochrome c oxidase concentration and tissue scattering during functional activation," Adv. Exp. Med. Biol. 737, 119-124 (2012).

24. A. Ghosh et al., "Reduction of cytochrome c oxidase during vasovagal hypoxia-ischemia in human adult brain: a case study," Adv. Exp. Med. Biol. 789(July), 463-467 (2013).

25. C. Kolyva et al., "Systematic investigation of changes in oxidized cerebral cytochrome c oxidase concentration during frontal lobe activation in healthy adults," Biomed. Opt. Express 3(10), 2550-2566 (2012).

26. C. Kolyva et al., "Cytochrome c oxidase response to changes in cerebral oxygen delivery in the adult brain shows higher brain-specificity than haemoglobin," Neuroimage 85, 234-244 (2014).

27. G. Bale et al., "Relationship between cerebral cytochrome-C-oxidase and oxygenation is associated with brain injury severity in birth asphyxiated infants," in Biomed. Opt. 2016, TM2B.4 (2016).

28. P. Phan et al., "Multi-channel multi-distance broadband near-infrared spectroscopy system to measure the spatial response of cellular oxygen metabolism and tissue oxygenation," Biomed. Opt. Express 7(11), 4424 (2016).

29. P. T. Phan et al., "Spatial distribution of changes in oxidised cytochrome c oxidase during visual stimulation using broadband near infrared spectroscopy imaging," Adv. Exp. Med. Biol. 923, 195-201 (2016).

30. G. Bale et al., "Oxygen dependency of mitochondrial metabolism indicates outcome of newborn brain injury," J. Cereb. Blood Flow Metab. 39(10), 2035-2047 (2018).

31. C. E. Cooper et al., "Measurement of cytochrome oxidase redox state by near infrared spectroscopy," Adv. Exp. Med. Biol. 413, 63-73 (1997).

32. C. E. Cooper and R. Springett, "Measurement of cytochrome oxidase and mitochondrial energetics by near-infrared spectroscopy," Philos. Trans. R. Soc. Lond. B. Biol. Sci. 352(1354), 669-676 (1997).
33. G. Bale et al., "A new broadband near-infrared spectroscopy system for in-vivo measurements of cerebral cytochrome-c-oxidase changes in neonatal brain injury," Biomed. Opt. Express 5(10), 3450-3466 (2014).

34. S. Mitra et al., "Pressure passivity of cerebral mitochondrial metabolism is associated with poor outcome following perinatal hypoxic ischemic brain injury," J. Cereb. Blood Flow Metab. 39(1), 118-130 (2019).

35. C. Kolyva et al., "Dependence on NIRS source-detector spacing of cytochrome c oxidase response to hypoxia and hypercapnia in the adult brain," Adv. Exp. Med. Biol. 789, 353-359 (2013).

36. I. de Roever et al., "Cytochrome-C-oxidase exhibits higher brain-specificity than haemoglobin in functional activation," in Biomed. Opt. 2016, p. BTh4D.4 (2016).

37. R. Zimmermann et al., "Silicon photomultipliers for improved detection of low light levels in miniature near-infrared spectroscopy instruments," Biomed. Opt. Express 4(5), 659 (2013).

38. F. B. Haeussinger et al., "Simulation of near-infrared light absorption considering individual head and prefrontal cortex anatomy: implications for optical neuroimaging," PLoS One 6(10), e26377 (2011).

39. D. Wyser et al., "Wearable and modular functional near-infrared spectroscopy instrument with multidistance measurements at four wavelengths," Neurophotonics 4(04), 1 (2017).

40. E. Wright, K. St. Lawrence, and M. Diop, "Conversion of a low cost offthe-shelf spectrometer into a suitable instrument for deep tissue spectroscopy," Proc. SPIE 8573, 85730V (2013).

41. M. Diop et al., "Improved light collection and wavelet de-noising enable quantification of cerebral blood flow and oxygen metabolism by a lowcost, off-the-shelf spectrometer," J. Biomed. Opt. 19(5), 057007 (2014).

42. R. Nosrati et al., "Event-related changes of the prefrontal cortex oxygen delivery and metabolism during driving measured by hyperspectral fNIRS," Biomed. Opt. Express 7(4), 1323 (2016).

43. P. Kaynezhad, "Miniature broadband-NIRS system to measure CNS tissue oxygenation and metabolism in preclinical research," Doctoral Thesis, University College London (2018).

44. R. H. Thiele et al., "Comparison of broadband and discrete wavelength near-infrared spectroscopy algorithms for the detection of cytochrome aa3 reduction," Anesth. Analg. 129, 1273-1280 (2018).

45. R. Nosrati et al., "Near infrared spectroscopy (NIRS) reveals the effect epinephrine on cerebral oxygen delivery and metabolism during cardiac arrest," in Biophotonics Congr.: Biomed. Opt. Congr. 2018 (Microsc./ Transl./Brain/OTS), p. BTh2C.6 (2018).

46. R. Nosrati et al., "Study of the effects of epinephrine on cerebral oxygenation and metabolism during cardiac arrest and resuscitation by hyperspectral near-infrared spectroscopy," Crit. Care Med. 47(4), e349-e357 (2019).

47. T. N. Nguyen et al., "Hyperspectral near-infrared spectroscopy assessment of the brain during hypoperfusion," J. Biomed. Opt. 24(03), 1 (2019).

48. D. T. Delpy et al., "Estimation of optical pathlength through tissue from direct time of flight measurement," Phys. Med. Biol. 33(12), 1433-1442 (1988).

49. S. Ijichi et al., "Developmental changes of optical properties in neonates determined by near-infrared time-resolved spectroscopy," Pediatr. Res. 58(3), 568-573 (2005).

50. B. Chance et al., "Phase modulation system for dual wavelength difference spectroscopy of hemoglobin deoxygenation in tissues," Proc. SPIE 1204, 481-491 (1990).

51. A. Duncan et al., "Optical pathlength measurements on adult head, calf and forearm and the head of the newborn infant using phase resolved optical spectroscopy," Phys. Med. Biol. 40(2), 295-304 (1995).

52. S. J. Matcher, M. Cope, and D. T. Delpy, "Use of the water absorption spectrum to quantify tissue chromophore concentration changes in nearinfrared spectroscopy," Phys. Med. Biol. 39(1), 177-196 (1994).

53. M. Essenpreis et al., "Wavelength dependence of the differential pathlength factor and the log slope in time-resolved tissue spectroscopy," Adv. Exp. Med. Biol. 333, 9-20 (1993).

54. A. Lorek et al., "Delayed ('secondary') cerebral energy failure after acute hypoxia-ischemia in the newborn piglet: continuous 48-hour studies by phosphorus magnetic resonance spectroscopy," Pediatr. Res. 36(6), 699-706 (1994).

55. L. Vanhamme, A. Van den Boogart, and S. Van Huffel, "Improved method for accurate and efficient quantification of MRS data with use of prior knowledge," J. Magn. Reson. 129, 35-43 (1997). 
56. O. A. Petroff et al., "Cerebral intracellular $\mathrm{pH}$ by $31 \mathrm{P}$ nuclear magnetic resonance spectroscopy," Neurology 35(6), 781-788 (1985).

57. J. W. Pettegrew et al., "Considerations for brain $\mathrm{pH}$ assessment by $31 \mathrm{P}$ NMR," Magn. Reson. Imaging 6(2), 135-142 (1988).

58. C. M. Florkowski, "Sensitivity, specificity, receiver-operating characteristic (ROC) curves and likelihood ratios: communicating the performance of diagnostic tests," Clin. Biochem. Rev. 29(suppl 1), S83-S87 (2008).

59. "HL-2000 family—ocean optics," https://oceanoptics.com/product/hl2000-family/ (accessed 14 May 2019).

60. F. Jobsis, "Noninvasive, infrared monitoring of cerebral and myocardial oxygen sufficiency and circulatory parameters," Science 198(4323), 1264-1267 (1977).

61. A. Bozkurt and B. Onaral, "Safety assessment of near infrared light emitting diodes for diffuse optical measurements," Biomed. Eng. Online 3(1), 9 (2004).

62. C. D. Strubakos et al., "Non-invasive treatment with near-infrared light: a novel mechanisms-based strategy that evokes sustained reduction in brain injury after stroke," J. Cereb. Blood Flow Metab. (2019).

63. T. I. Karu, "Mitochondrial signaling in mammalian cells activated by red and near-IR radiation," Photochem. Photobiol. 84(5), 1091-1099 (2008).

64. A. P. Sommer et al., "Biostimulatory windows in low-intensity laser activation: lasers, scanners, and NASA's light-emitting diode array system," J. Clin. Laser Med. Surg. 19(1), 29-33 (2001).
65. M. Hüttemann et al., "Regulation of mitochondrial respiration and apoptosis through cell signaling: cytochrome c oxidase and cytochrome $\mathrm{c}$ in ischemia/reperfusion injury and inflammation," Biochim. Biophys. Acta 1817(4), 598-609 (2012).

66. M. Caldwell et al., "BrainSignals revisited: simplifying a computational model of cerebral physiology," PLoS One 10(5), e0126695 (2015).

67. G. E. Strangman, Z. Li, and Q. Zhang, "Depth sensitivity and sourcedetector separations for near infrared spectroscopy based on the Colin27 brain template," PLoS One 8(8), e66319 (2013).

68. I. de Roever et al., Functional NIRS Measurement of Cytochrome-COxidase Demonstrates a More Brain-Specific Marker of Frontal Lobe Activation Compared to the Haemoglobins, pp. 141-147, Springer, Cham (2017).

69. S. Gunadi and T. S. Leung, "Spatial sensitivity of acousto-optic and optical near-infrared spectroscopy sensing measurements," J. Biomed. Opt. 16(12), 127005 (2011).

70. G. Bale, "Development of optical instrumentation and methods to monitor brain oxygen metabolism: application to neonatal birth asphyxia," Doctoral Thesis, University College London (2016).

71. M. Cope, "The application of near infrared spectroscopy to non invasive monitoring of cerebral oxygenation in the newborn infant," Doctoral Thesis, University College London (1991).

Biographies of the authors are not available. 